\title{
Coupling of cytoplasm and adhesion dynamics determines cell polarization and locomotion
}

\author{
Wolfgang Alt Martin Bock $^{2} \quad$ Christoph Möhl ${ }^{3}$
}

October 24, 2018

\begin{abstract}
Observation of epidermal cells or cell fragments on flat adhesive substrates has revealed two distinct morphological and functional states: A non-migrating symmetric "unpolarized state" and a migrating asymmetric "polarized state". They are characterized by different spatial distributions and dynamics of important molecular components as F-actin and myosin-II within the cytoplasm, and integrin receptors at the plasma membrane contacting the substratum, thereby inducing so-called focal adhesion complexes. So far, mathematical models have reduced this phenomenon either to gradients in regulatory control and signaling molecules or to different mechanics of the polymerizing and contracting actin filament system in different regions of the cell edge.

Here we offer an alternative self-organizational model in order to reproduce and explain the emergence of both functional states for a certain range of dynamical and kinetic model parameters. We apply an extended version of a two-phase, highly viscous cytoplasmic flow model with variable force balance equations at the moving edge, coupled to a 4-state reaction-diffusion-transport system for the bound and unbound integrin receptors beneath the spreading cell or cell fragment. In particular, we use simulations of a simplified 1-D model for a cell fragment of fixed extension to demonstrate characteristic changes in the concentration profiles for actin, myosin and doubly bound integrin, as they
\end{abstract}

${ }^{1}$ corresponding author, eMail wolf .alt@uni-bonn.de

${ }^{2}$ Universität Bonn, Theoretische Biologie, Kirschallee 1-3, 53115 Bonn, Germany

${ }^{3}$ Forschungszentrum Jülich, Institut für Bio- und Nanosysteme (IBN),

Institut 4: Biomechanik (IBN4), 52425 Jülich, Germany 
occur during transition from the symmetric stationary state to the polarized migrating state. In the latter case the substratum experiences a low magnitude "pulling force" within the larger front region, and an opposing high magnitude "disruptive force" at the shorter rear region. Moreover, simulations of the corresponding 2-D model with free boundary show characteristic undulating protrusions and retractions of the cell (fragment) edge, with local accumulation of doubly bound adhesion receptors behind it, combined with a modulated retrograde F-actin flow. Finally, for a stationary model cell (fragment) of symmetric round shape, larger fluctuations in the circumferential protrusion activity and adhesion kinetics can break the radial symmetry and induce a gradual polarization of shape and concentration profiles, leading to continuous migration in direction of the leading front.

The aim of the chapter is to show how relatively simple laws for the smallscale mechanics and kinetics of participating molecules, responsible for the energy consuming steps as filament polymerization, pushing and sliding, binding and pulling on adhesion sites, can be combined into a non-linearly coupled system of hyperbolic, parabolic and elliptic differential equations that reproduce the emergent behavior of polarization and migration on the large-scale cell level.

\section{Contents}

1 Biology of cell polarization and migration 3

1.1 Asymmetry of actin polymerization and substrate adhesion . . . . . . 3

1.2 Flow of actin filaments and myosin gradient . . . . . . . . . . . . 4

2 Previous models of cytoplasm and adhesion dynamics 5

3 Multiply coupled reaction-diffusion-flow model 7

3.1 Mass balance and flow equations . . . . . . . . . . . . 8

3.1.1 Mass conservation for the two phases cytoskeleton and cytosol. . . . 9

3.1.2 Reaction-transport-diffusion equations for myosin oligomers. . . . . . 10

3.1.3 Mass conserving flow of the dorsal plasma membrane. . . . . . . . 11

3.1.4 Reaction-transport-diffusion equations for membrane integrins. . . . 12

3.1.5 Mass flux conditions at the free boundary. . . . . . . . . . . 13

3.2 Force balance equations . . . . . . . . . . . . . . . . 15

3.2.1 Two-phase flow equations for cytoskeleton and cytosol. . . . . . . . 15

3.2.2 Stress and pressure balance conditions at the free boundary. . . . . . 16

3.2.3 Boundary pressure functions at the cell edge. . . . . . . . . . 18

3.2.4 Global force balance at the adhesive substratum. . . . . . . . . 20 
4 Results of model simulations

4.1 Spontaneous cell polarization in the 2 -D model . . . . . . . . . . . . 20

4.2 Induced onset of cell polarization and migration in the 1-D model . . . . . 23

4.3 Migration speed in a simplified 1-D model . . . . . . . . . . . 30

\section{Discussion and outlook to further modeling}

\section{Biology of cell polarization and migration}

Cell polarization and migration plays a central role in the development and maintenance of tissues in multicellular organisms. During ontogenesis, new tissues are formed by the coordinated division and locomotion of single cells. The polarization of a cell not only defines the direction of migration [30] but also the cell division axis [61] and thus the three-dimensional structure of tissues, organs and finally the whole organism.

\subsection{Asymmetry of actin polymerization and substrate adhesion}

The coordinated development and release of focal adhesions (FAs) is a basic requirement for directed cell migration. Migrating cells feature pronounced adhesion dynamics and a structural polarity with a clearly distinguishable frontal and rear area. Actin polymerization predominates at the cell front resulting in a protruding lamella in direction of migration. New focal adhesions composed of clustered protein complexes develop at the lower membrane of the lamella near the leading edge and couple the F-actin-network mechanically to extracellular matrix proteins. Simultaneously, the matured FAs residing at the opposed trailing edge are dissolved while myosin driven contraction of F-actin moves the cell body forward [10, 36].

Arising from the process described above, the polarity of the cell can be referred to two structural asymmetries, which are key requirements for effective cell migration: asymmetry of actin polymerization and asymmetry of adhesion strength. The growth of actin filaments has to predominate at the cell front for pushing the leading edge in direction of migration [63, 58]. To move the cell body forward, it has to be released from the substrate during contraction by dissolving the FAs at the back (rear release) while the FAs at the cell front have to remain stable to provide a mechanical attachment for the contractile machinery pulling the cell body. In absence of rear release, traction forces could be dominated by adhesion forces and the cell would get stuck [54]. In this regard, the spatial distribution of adhesion strength and actin polymerization defines the direction of migration and could be specifically regulated due to directed cell movement. 

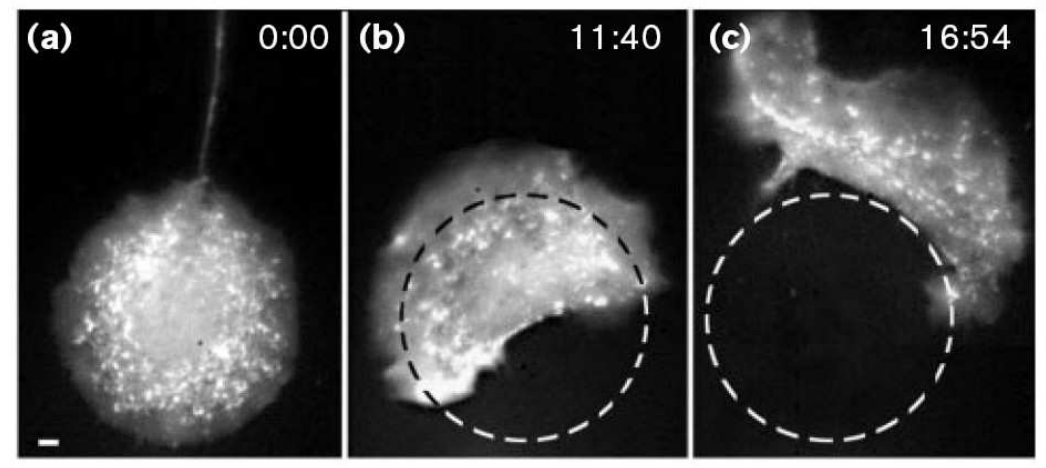

Figure 1: Cell fragment experiments where the mechanical stress (release) induces a transition from a circular non-migrating state (a) to a polarized migrating state (b,c). The cell fragment was labelled with fluorescent myosin II. Time scale is min:sec and length scale of bar is $2 \mu \mathrm{m}$ (from [63]).

\subsection{Flow of actin filaments and myosin gradient}

The mechanisms underlying the structural polarity of migrating cells are still under discussion, particularly what concerns an intrinsic directionality of the cytoplasm. For example, the finding that asymmetric adhesive structures define polarization of a touching cell [30] suggests that directional flow of the actin cytoskeleton is involved in the process of cell polarization. Moreover, recent studies on fish keratocytes have revealed that polarization occurs spontaneously and is accompanied by a reorganization of the actin cytoskeleton, which finally leads to cell locomotion 64. In these experiments, unpolarized solitary cells feature a circular shape with a radially symmetric actin distribution. Although the cells do not move, transient protrusions and retractions appear at the cell edge, and actin flows centripetally with a decreasing flow gradient from the cell edge to the center. In this apparently unstable state spontaneous symmetry breaking results in a faster inward flow and in an increased concentration of actin at the rear region of the cell. On the opposed front edge, the reduced inward actin flow causes protrusion and the development of a lamellipodium. In this polarized state, the cell starts to migrate while attaining a more or less constant shape.

A similar behavior was observed with cell fragments extracted from the lamella of fish keratocytes [63], see Fig. 1. Since these fragments were lacking most cell organelles and the microtubule system, they were mainly limited to the actin-myosin machinery. They appeared either in an unpolarized, non-migrating state with homogeneous actin-myosin distribution or a polarized, migrating state with a rising myosin concentration from front to rear. Interestingly, polarization of fragments could be induced by mechanical stimulation, e.g. by shear flow or stress release leading to a transition from the stationary to the 
migrating state.

Cell polarization is discussed to result from the local activation of GTPases by the rhofamily [62, 54, 43. These proteins are known to regulate actin polymerization, myosin activity and FA assembly, whereas it remains unclear how the distribution of the GTPases is controlled. Recent experiments have revealed that by inhibition of myosin activating signalling pathways the cell's ability to polarize is reduced [64]. However, the observations of cells or cell fragments to polarize either spontaneously or due to mechanical perturbations, raise doubts that biochemical signalling is the primary reason for inducing and maintaining polarity. Experiments focussing on cell mechanics rather show that direction and strength of locomotion forces are inherently connected with the retrograde F-actin flow [53, 22]. This suggests that the mechanical action of myosin, namely to induce cytoplasmic

contraction, flow and force transduction at adhesive sites, plays a key role in explaining the ubiquitously observed phenomena of cell polarization and locomotion.

\section{Previous models of cytoplasm and adhesion dynamics}

The first detailed mathematical model coupling cytoskeleton and adhesion dynamics was developed by Lauffenburger and coworkers almost two decades ago [17. Here, the contractile actin-myosin network and its interaction with bound adhesion sites is represented as a mechanical system of connected viscoelastic units of generalized Kelvin-Voigt type, which constitute the (three) inner segments as well as a front segment (lamellipod) and a tail segment (uropod) for a rectangular model cell of fixed width and length. A coupled system of reaction-diffusion equations for free and substrate bound integrins or adhesion receptors on the dorsal (lower) and ventral (upper) part of the model cell is solved under pseudo steady-state assumptions. This dynamically provides the number of adhesion bonds in both end segments, lamellipod and uropod, whereby their rupture (dissociation) kinetics exponentially increases with force load onto a bound adhesion site. The resulting non-linear dynamics for the local (forward or backward) displacement of each viscoelastic unit produces a persistent forward translocation of the whole model cell. However, this is achieved only if a front-tail asymmetry is presupposed, either by exposing more free adhesion receptors, or by assuming higher adhesion bond affinity at the front compared to the tail. The authors present a series of results on how the simulated cell migration speed depends on various model parameters as, for example, cytoskeletal contractility or adhesion strength. A later variant of this model presents a more explicit study of adhesion bond disruption kinetics at the rear of the cell and already uses a four-state model for integrin binding to the cytoskeleton and to the substratum [51].

In recent years a series of more elaborate models have been developed, accounting for details of the meanwhile discovered molecular regulation mechanisms for the chemical and mechanical processes, particularly at the free boundary of a moving cell. One model type is based on spatially discrete algorithms (cellular Pott's model) using the definition of local 
energies to determine the protrusion and retraction of boundary elements via the stochastic Metropolis rule, e.g. [39, 46, 47].

Another class of mechanical cell models takes into account branching and anisotropy of cytoskeletal actin filaments at various times and in various regions of the cell, see e.g. 65, 23, 28. Besides explicit constructions of an elastically cross-linked network in the leading edge [48, 32] biophysical models have been developed to capture different dynamics of Arp2/3induced branching and myosin-induced contraction of F-actin networks by Mogilner and coworkers [44, 25, 33]. The last article is particularly devoted to explain the mentioned polarization experiments with cell fragments (see Fig. 1) by assuming a different actinmyosin network organization at the rear in comparison to the front region.

Based on the 'reactive flow' model by Dembo and coworkers [13, 12, 3], the most elaborate model extension has been presented by Oliver et al. [49]: they use full 3-D two-phase flow equations with free ventral boundary and with two additional rapidly diffusing messenger concentrations that regulate actin network contractility and (de-)polymerization. Moreover, cell adhesion is modeled by a Navier-slip boundary condition at the substratum, in which only constant adhesive properties are taken into account. The analysis of this complex model, being performed in the thin film limit, is restricted to linear-stability arguments for ruffle generation and to local expansion analyses at the moving tip; there, phenomenological equations for boundary mass fluxes are considered without specifying the types of molecular mechanisms for tip protrusion. Finally, quantitative estimations for the pseudopod protrusion and cell translocation speed under various sub-limit assumptions are given, which turn out to be consistent with observed values, particularly for osteoblasts, though no numerical simulations are given that would re-insure the analytic results. A similar thin-film approximation of the 2-D equations under incompressibility assumptions for a 'viscous polar gel' has been used in [34] to derive explicit expressions for the advancing speed of a cell lamella, but again by predefining its polarity.

Except the last one, all so-far mentioned models do not explicitly quantify the varying force field, which is applied by the cytoskeleton onto the substrate covered by a migrating cell and which has been approximately reconstructed by different inverse methods from experimental assays of cells, e.g. moving on flexible substrata [15, 38, 56, 40, 6]. A first model implementing force transduction to the substrate has been proposed by Gracheva and Othmer 24 by specifying a spatially one-dimensional system of viscoelastic equations for cytoskeleton dynamics, whose polymerization, contractility and adhesive binding is regulated by signalling molecules. However, they make a pseudo-steady-state assumption for the binding kinetics of myosin polymers to actin filaments, and of transmembrane integrin proteins to the substratum. Moreover, they impose artificially defined gradients from tail to front of certain regulatory proteins in order to stimulate polarized cell translocation.

Recently, adhesion kinetics has also been implemented into an extended cytoplasm flow model [4, 5] describing the F-actin dynamics in an annular domain and its coupling to lamellipodial protrusions and retractions [59]. Force dependent maturation of FA complexes and active polymerization of $\mathrm{F}$-actin enable the simulation model to reproduce characteristics 
of fibroblast shape deformation and translocation.

In an earlier publication we have presented another extension of the basic two-phase flow model for the 2-dimensional viscous cytoplasm dynamics [2] by coupling the constitutive hyperbolic-elliptic equations to a system of four reaction-diffusion-transport equations for the integrins beneath the cell or cell fragment [35]. Here we propose a generalized continuum model, in which we couple cytoplasm and adhesion dynamics with mechanical tension and transport of the plasma membrane, in order to reproduce spontaneous and induced cell polarization leading to migration, with assembly of adhesion sites at the cell front and adhesion release at the cell's rear end. Moreover, the model exhibits typical features of migrating cells as protrusion/retraction cycles, rearward actin flow, pulling forces at the front and a concentration of disruptive forces at the rear. In the model, the cytoplasm is described as a viscous and contractile fluid of polymers representing the actin cytoskeleton interpenetrated by an aqueous phase. This actin filament network is preferentially assembled at the cell edge, and can be contracted by cross-linking with diffusing myosin oligomers. The moving actin filaments then couple to transmembrane adhesion proteins that are freely diffusing in the membrane or bound to the substrate on the extracellular domain. Thus, the cytoskeleton mechanically connects to the substrate through dynamic binding processes which results in force transduction and finally cell locomotion.

Throughout our model presentation we rely on continuum descriptions, in which macroscopic mass and momentum laws are combined with mesoscopic submodels for fast molecular kinetics due to adequate pseudo-steady-state assumptions.

\section{Two-phase flow model for cytoplasm coupled to reaction, diffusion and transport for myosin-II and integrin proteins}

For simplicity, we restrict our model derivation and analysis to a flat two-dimensional geometry, so that cells or cell fragments are assumed to be homogeneously spread on the substrate without considerable change of cell height. Thereby, three-dimensional effects around the cell nucleus (e.g. due to cell rolling) or along the ventral (upper) plasma membrane are neglected. In order to reproduce the main biophysical mechanisms and biochemical processes that enable a cell to polarize and translocate on an adhesive substratum, we nevertheless distinguish between the cytoplasm and the exterior plasma membrane: On the one hand, F-actin assembly, myosin kinetics and viscous network flow take place within the cytoplasmic interior of the cell. On the other hand, integrin binding to substrate or cytoskeleton and its transport or diffusion are confined to the dorsal (lower) plasma membrane, where forces are transduced to or generated at the cell periphery, leading to tip protrusion or retraction. Thus, the moving cell (fragment) is simply represented by a time-dependent connected domain $\Omega(t) \subset \mathbb{R}^{2}$, over which the cytoplasmic volume extends with fixed constant height, and any ruffles or blebs on top of the cell are neglected. We rather assume that the cell dynamics is completely determined by a flat cytoskeleton 


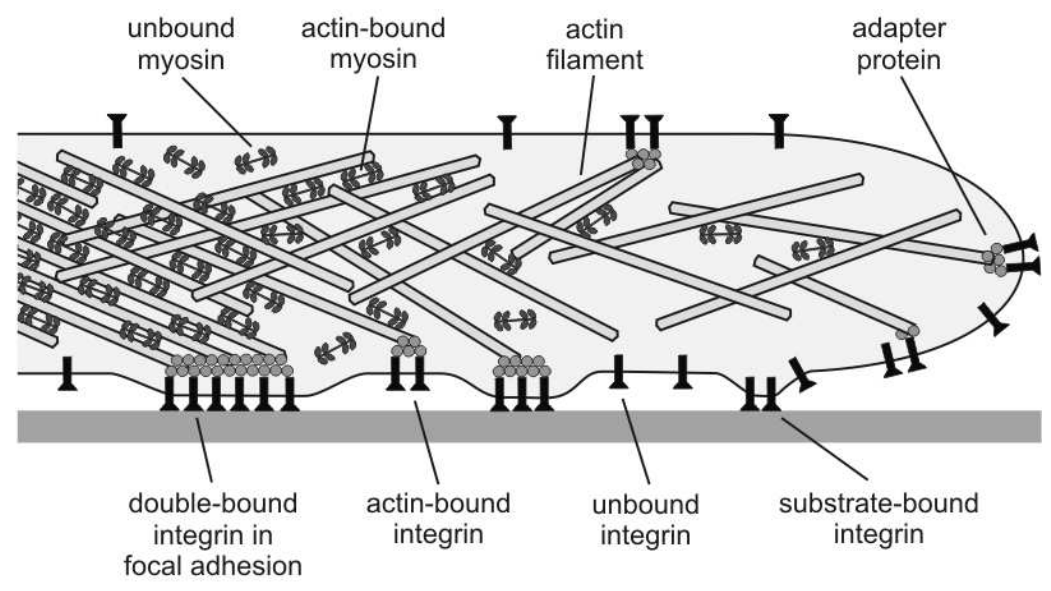

Figure 2: Schematic longitudinal section through a cell fragment (or a cell lamella) of constant height with involved proteins. For further explanation and model derivation see the following sections.

sheet of F-actin network, see Fig. 2. This cross-linked filament phase with volume fraction $\theta(t, \mathbf{x}), \mathbf{x} \in \Omega(t)$ is of constant thickness and connected to the upper and lower plasma membrane in such a way that also the cytosol, i.e. the solvent phase with volume fraction $(1-\theta(t, \mathbf{x}))$, is confined to the same volume space.

By suitable model simplifications, we aim to capture the self-organizational power of the cytoplasm as a two-phase fluid coupled with the reaction, transport and diffusion of a series of chemical ingredients. We explicitly model the kinetics and dynamics of only those F-actin associated proteins that induce the main bio-mechanical processes of force generation and transduction to the substratum, namely myosin oligomers and transmembrane integrin proteins. All other regulatory proteins such as e.g. Rac, Rho or the branching protein Arp2/3 or smaller substrate molecules, e.g. monomeric G-actin, are assumed to rapidly diffuse within the cytosol under fast regeneration, so that they attain constant reservoir concentrations serving as parameters in the model.

\subsection{Mass balance and flow equations}

In the chosen two-dimensional continuum description, cell deformation and translocation with respect to the fixed substratum is represented by movement of the cell edge $\Gamma(t)=\partial \Omega(t)$ which, however, is induced by three distinguished mass flows with potentially different transport velocity fields on $\bar{\Omega}$, namely the mean velocities $\mathbf{v}$ of the F-actin cytoskeleton $(\theta)$ and $\mathbf{w}$ of the aqueous cytosol $(1-\theta)$ plus the transport velocity $\mathbf{u}$ of the lower dorsal plasma membrane. Thereby both $\mathbf{v}$ and $\mathbf{w}$ are averaged over the constant cell height. For the membrane area flux $\mathbf{u}$ we make the simplifying assumption that the 
lipid bilayer between the flat substratum and an adhering cell is always stretched out to its maximal extension with constant area concentration of the lipid-protein mixture, thus constituting an incompressible two-dimensional Newtonian fluid. Then, freely diffusing transmembrane integrin proteins are additionally transported by the membrane velocity $\mathbf{u}$, myosin oligomers by the cytosol velocity $\mathbf{w}$, and F-actin bound myosin-II motor molecules by the cytoskeleton velocity $\mathbf{v}$. The detailed mass balance equations are discussed in the following paragraphs.

\subsubsection{Mass conservation for the two phases cytoskeleton and cytosol.}

Cytoskeleton and cytosol in the flat two-dimensional geometry can experience counteracting flows due to local contraction, assembly or relaxation of the F-actin network, while the bulk cytoplasmic fluid with constant volume fraction 1 can be assumed to be incompressible, at least in the range of occurring pressure differences $\lesssim \mathrm{kPa}$. Compare the analogous situation of a water-filled sponge that is internally condensing without changing its shape. Then, because of the fixed height assumption, the total two-dimensional volume flux $\mathbf{W}=$ $\theta \mathbf{v}+(1-\theta) \mathbf{w}$ is divergence free at any time $t$, yielding the first local mass conservation law

$$
\nabla \cdot(\theta \mathbf{v}+(1-\theta) \mathbf{w})=0
$$

Therefore, also the total cell volume (i.e. the 2-dimensional cell area) is conserved over time, so that the 'bulk' fluid moves together with the free boundary $\Gamma(t)$, meaning that the total volume flux $\mathbf{W}$ has to fulfill the natural free boundary condition

$$
\nu_{\Gamma} \cdot \mathbf{W}=\dot{\Gamma}
$$

with $\nu_{\Gamma}$ denoting the exterior normal of $\Gamma$ and $\dot{\Gamma}$ quantifying the normal speed of the cell edge.

In addition to possible convection, the F-actin network can locally be assembled by filament polymerization from the pool of monomeric G-actin within the cytosol and it can be disassembled by the reverse process of severing or depolymerization. This mass exchange between the two phases is given by a second conservation law, which for the filament volume fraction $\theta$ can be written as

$$
\partial_{t} \theta+\nabla \cdot(\theta \mathbf{v})=R(\theta)
$$

with a net assembly rate $R(\theta)$ to be modeled. According to our simplifying assumption, the dependence on G-actin, Arp2/3 and possible regulatory proteins enters only via constant parameters:

$$
R(\theta)=\left(k_{\mathrm{on}} a_{g}-k_{\mathrm{off}}\right) B(\theta)-r \theta
$$

with concentration of globular actin $a_{g}$, polymerization rate $k_{\text {on }}$ and depolymerization rate $k_{\text {off }}$ at the filaments' plus (barbed) ends, as well as another lumped disassembly rate $r$, 
see e.g. [42]. Due to relatively fast nucleation and capping of actin filaments, the relative number $B$ of barbed ends is assumed to stay in pseudo-equilibrium with the local F-actin concentration $a=\theta \cdot a_{\max }$, where we suppose a maximal condensation of actin filaments in the order of $a_{\max }=800 \mu \mathrm{M}$. Following [42] we write

$$
B(\theta)=\frac{1}{\omega}\left(\varepsilon+\nu_{0} \frac{\theta}{K_{a} / a_{\max }+\theta}\right)
$$

with capping rate $\omega$, a basic nucleation rate $\varepsilon$ and an induced branching rate $\nu_{0}=\nu_{a} \operatorname{Arp} 0$, proportional to the concentration Arp0 $[\mu \mathrm{M}]$ of activated Arp2/3, together with a halfsaturation concentration $K_{a}$ for its primary actin binding site.

\subsubsection{Reaction-transport-diffusion equations for myosin oligomers.}

Myosin-II oligomers are the most important actin binding proteins that are responsible for the generation of contractile forces within cross-linked F-actin networks. Thus, their spatial distribution within a polarizing or moving cell plays a key role for the cytoplasm dynamics. In a most simple way we only distinguish between freely diffusing myosin oligomers $\left(m_{f}\right)$ and those that are bound to cytoskeletal actin filaments $\left(m_{b}\right)$ and, therefore, are convected with velocity $\mathbf{v}$. Since free myosin tetramers first have to attach to the actin network at one binding site, we consider this bimolecular reaction $\left(m_{f}\right.$ with $\left.a\right)$ as the rate limiting step. Correspondingly we assume, that the relatively faster processes of double binding $\left(m_{b}\right.$ with a) and power stroke formation are in a pseudo-steady state. Thereby the contractile stress $\psi=\psi_{0} m_{b} \theta$ with $\theta=a / a_{\max }$ in the network is determined, see equation (25) in the following section. Finally, we suppose a constant diffusivity $D_{m}$ for free myosin oligomers, embedded into the cytosol flow w. Under these assumptions the local mass balance equations for the corresponding myosin concentrations are:

$$
\begin{aligned}
\partial_{t} m_{f} & =\nabla \cdot\left(D_{m} \nabla m_{f}-m_{f} \mathbf{w}\right)-\alpha_{m} \cdot a \cdot m_{f}+\delta_{m}(a) \cdot m_{b} \\
\partial_{t} m_{b} & =-\nabla \cdot\left(m_{b} \mathbf{v}\right)+\alpha_{m} \cdot a \cdot m_{f}-\delta_{m}(a) \cdot m_{b} .
\end{aligned}
$$

For the association rate $\alpha_{m}$ we assume a constant parameter, whereas the dissociation rate is supposed to increase quadratically with increasing network concentration $a$ because of steric inhibition or competition for binding sites:

$$
\delta_{m}(a)=\delta_{m}^{0}\left(1+\frac{a^{2}}{a_{\mathrm{opt}}^{2}}\right)=\delta_{m}^{0}\left(1+\frac{\theta^{2}}{\theta_{\mathrm{opt}}^{2}}\right)
$$

with an optimal actin network concentration $a_{\mathrm{opt}}=a_{\max } \theta_{\mathrm{opt}}$. Moreover, in the case of fast diffusion and no transport, the constant equilibrium concentrations $m_{f}^{*}$ and $m_{b}^{*}$ satisfy the equality

$$
m_{b}^{*}=\frac{\alpha_{m} a}{\delta_{m}(a)} m_{f}^{*}
$$

so that the resulting contractile stress $\psi(\theta)$ becomes optimal for $\theta=\theta_{\text {opt }}$, Fig. 5 below. 


\subsubsection{Mass conserving flow of the dorsal plasma membrane.}

As mentioned before, we will assume that the dorsal plasma membrane beneath an adhering cell (fragment) is stretched and that small fluctuations can be neglected. On the contrary top side, the ventral plasma-membrane usually shows extensive wrinkles or folds, which most probably are induced by the contractile action of the cytoskeleton itself, indicating that the plasma membrane tip at the cell edge stays under a positive tension $\tau^{\Gamma}$. Before discussing the corresponding dynamics of the dorsal membrane, we state the two extreme possibilities, namely whether the membrane moves together with the cell or not:

1. Membrane sticking to substrate: During cell translocation over the substratum the whole dorsal membrane stays fixed and no slip may occur due to relatively strong interaction forces with the substratum, e.g. via the glykocalix, so that $\mathbf{u} \equiv 0$.

2. Membrane sticking to cell edges: The dorsal membrane is pulled along the substratum due to cytoplasm protrusions at the leading front, but with no slip around the lamellar tips due to strong membrane curvature. In this way the membrane area flux satisfies the mass conservation law together with a boundary condition analogous to (2), namely

$$
\nabla \cdot \mathbf{u}=0 \text { on } \Omega(t) \quad \nu_{\Gamma} \cdot \mathbf{u}=\dot{\Gamma} \text { on } \Gamma(t) .
$$

In the latter case, the membrane can slip over the substratum, thereby experiencing a finite frictional drag force, see (40) below, whereas in the first case this force is infinitely large to suppress slipping - but then the membrane has to slip around the tips at moving cell edges. Clearly, a certain mixture of both possibilities is physically realizable but not considered here.

Moreover, frictional drag onto the dorsal membrane can also occur at its cytoplasmic side. Whereas the relative flow of cytosol will have negligible effects, the horizontal F-actin flow develops a vertical flow profile. This profile depends on the amount of 'Navier-slip' in a cortical layer on top of the dorsal membrane and emerges due to frictional forces between actin filaments and membrane proteins. In analogy to the more explicit thin film approximation [49] we only consider the averaged profile velocity $\mathbf{v}$. In this way we can introduce a simplifying cortical slip parameter, $0<\kappa<1$, so that the effective relative velocity between cytoskeleton and dorsal membrane is reduced to $\kappa(\mathbf{v}-\mathbf{u})$. Thus, the resulting effective velocity of cortical F-actin in a thin layer above dorsal membrane and substratum is given by

$$
\mathbf{v}_{c}=\kappa \mathbf{v}+(1-\kappa) \mathbf{u} .
$$

Here the factor $\kappa$, which clearly depends on the viscous shear properties of the cytoskeleton, should be larger than 0.5 to reflect observations of a generally slippy behavior (see e.g. 29, 22]). Moreover, we suppose that the vertical profile, thus $\mathbf{v}_{c}$, is not changed if some of the cortical actin filaments are (transiently) bound to integrins in the dorsal membrane, see 


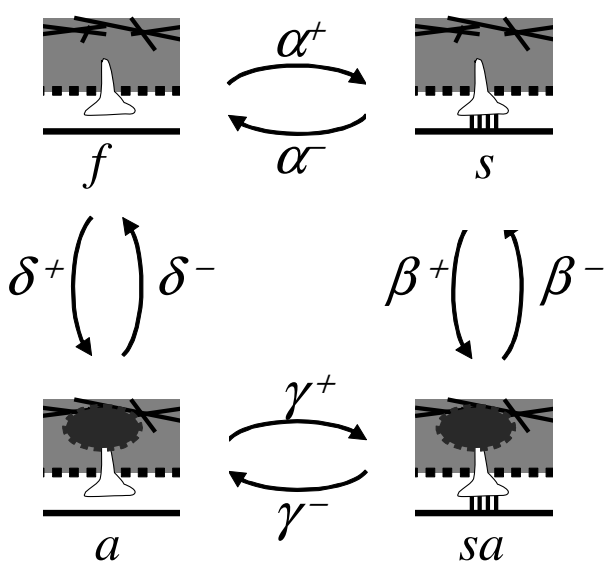

Figure 3: Scheme of four states of the transmembrane adhesion protein integrin. $(f)$ : unbound and freely diffusing within the dorsal membrane, $(s)$ : bound to the substrate, $(a)$ : bound to the actin network and thus moving with the actin-flow, $(s a)$ : bound to actin and substrate (force-transducing state). Integrins can switch between these states due to reversible binding kinetics with binding/unbinding rates $\alpha, \beta, \gamma$ and $\delta$.

Fig. 3 and the following paragraph: Either those bound integrins or integrin complexes are passively pulled through the lipid-protein bilayer with relative velocity $\mathbf{v}_{c}-\mathbf{u}$ or, in case of substrate-fixed adhesion bonds, with relative velocity $-\mathbf{u}$. The whole F-actin network above such a bond is slowed down by a certain local frictional force per adhesion site,

$$
\mathbf{F}_{c}=\Phi_{0} \theta \mathbf{v}_{c},
$$

entering into the corresponding force balance law, see $(23)$ below. In any case, the frictional drag onto the dorsal membrane, induced by the relative motion of singular integrin complexes, will be neglected in our model.

\subsubsection{Reaction-transport-diffusion equations for membrane integrins.}

The mechanical connection between the actin cytoskeleton and extracellular matrix proteins is provided by transmembrane integrins appearing in four different states [51, 35], see Fig. 3 .

In the freely diffusing state $f$, integrins are neither coupled to the substrate nor to the actin cytoskeleton and move according to a simple diffusion-transport law within the dorsal membrane. These integrins can change their state by binding to the actin cytoskeleton $(a)$ or to the substrate $(s)$. In state $a$, the integrins move with the cortical F-actin velocity $\mathbf{v}_{c}$, whereas integrins in state $s$ remain stationary with respect to the substrate. Actin- or substrate-bound integrins can switch back into the freely diffusing state $f$ by unbinding, 
or into the state $s a$ by coupling to the cytoskeleton and the extracellular matrix. Cell adhesion occurs only in this double bound state $s$ representing a focal adhesion (FA), where the frictional force $\mathbf{F}_{c}(12)$ of the moving actin-network is transduced to the substrate. The concentration of integrins $c_{\#}$ in the different states is described by the following coupled system of differential equations consisting of terms for spatial movement and binding kinetics.

$$
\begin{aligned}
\partial_{t} c_{f} & =\nabla \cdot\left(D_{f} \nabla c_{f}-c_{f} \mathbf{u}\right)+\alpha^{-} c_{s}+\delta^{-} c_{a}-\left(\delta_{0}^{+} a+\alpha^{+}\right) c_{f} \\
\partial_{t} c_{a} & =-\nabla \cdot\left(c_{a} \mathbf{v}_{c}\right)+\delta_{0}^{+} a c_{f}-\left(\delta^{-}+\gamma^{+}\right) c_{a}+\gamma_{0}^{-} \exp \left(\rho_{\gamma}\left|\mathbf{F}_{c}\right|\right) c_{s a} \\
\partial_{t} c_{s} & =\alpha^{+} c_{f}-\left(\alpha^{-}+\beta_{0}^{+} a\right) c_{s}+\beta_{0}^{-} \exp \left(\rho_{\beta}\left|\mathbf{F}_{c}\right|\right) c_{s a} \\
\partial_{t} c_{s a} & =\gamma^{+} c_{a}+\beta_{0}^{+} a c_{s}-\left(\beta_{0}^{-} \exp \left(\rho_{\beta}\left|\mathbf{F}_{c}\right|\right)+\gamma_{0}^{-} \exp \left(\rho_{\gamma}\left|\mathbf{F}_{c}\right|\right)\right) c_{s a}
\end{aligned}
$$

The bonds of the force-transducing integrins $\left(c_{s a}\right)$ can break when they experience a mechanical stress by the cytoskeleton, in our model given by the modulus of the force vector $\mathbf{F}_{c}$ defined in (12). Then, according to the theory of Bell [7, 57] the dissociation rates $\gamma^{-}$ and $\beta^{-}$depend exponentially on the mechanical load $\left|\mathbf{F}_{c}\right|$, see equations $(14,16)$ above, with $\gamma_{0}^{-}$and $\beta_{0}^{-}$describing the basic dissociation rates without load and the exponential coefficients $\rho_{\#}=\zeta_{\#} / k_{B} T$ measuring potentially different rupture rates from the substrate or cytoskeleton binding site, respectively.

\subsubsection{Mass flux conditions at the free boundary.}

In addition to the already mentioned bulk flux conditions (2) and (11) related to the normal speed of the cell edge $\Gamma(t)$, we have to impose compatible boundary conditions onto the concentrations of those molecular species that are not fixed to the substratum. For the two parabolic diffusion equations (6) and (13) we impose, respectively, natural zeroflux boundary conditions onto freely diffusing myosin $\left(m_{f}\right)$ and fixed Dirichlet conditions $\left(c_{f}=c_{f}^{0}\right)$ onto freely diffusing integrin. Thereby we suppose a constant reservoir of fresh adhesion receptors expressed in the upper ventral membrane and diffusing (or eventually being transported) from there around the lamellar tip into the lower dorsal part of the membrane.

The F-actin flux $\theta \mathbf{v}$ cannot leave the cell, meaning that on the free boundary $\Gamma$ the relative inward normal $F$-actin velocity always has to satisfy the inequality

$$
V=\dot{\Gamma}-\nu_{\Gamma} \cdot \mathbf{v} \geq 0
$$

If the strong inequality $V>0$ holds at a certain boundary point, then two different modeling situations may arise for the cell edge:

1. No-stick condition at the lamellar tip: Local disruption of the actin network from the edge is allowed, e.g. under suitable load conditions [35], so there is no new Factin production directly at plasma membrane edge. Therefore we have to impose 
zero-Dirichlet condition for the F-actin concentration

$$
\theta=0 \text { if } V>0 \text { holds at a non-sticky point } \mathbf{x} \in \Gamma \text {. }
$$

2. Network sticking to the lamellar tip: As indicated in Fig. 4, active polymerization of actin filaments directly at the plasma membrane is allowed either (a) at fluctuating free filament ends touching the tip membrane [43], or (b) at filaments that are bound to clamp-motor proteins anchored in the tip membrane [16]. Both cases could occur simultaneously in a local region, but whether active polymerization with inward mass flux $\theta V>0$ can take place depends on local force balance conditions, see Section 3.2 and (36) below.

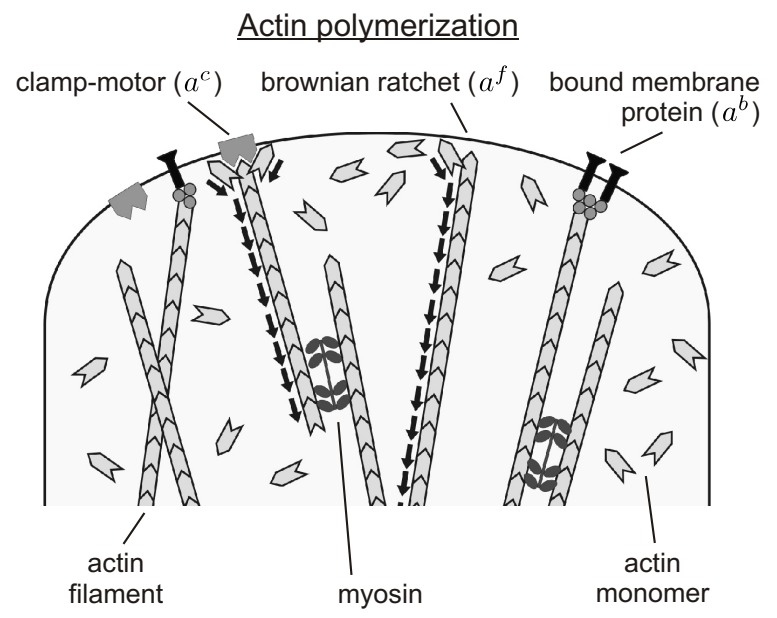

Figure 4: Schematic top view onto the lamellar tip of a cell (fragment) showing three possibilities for actin filament ends to interact with the free plasma membrane: anchoring at a membrane protein, as integrin; freely fluctuating with polymerization by a "Brownian ratchet" mechanism; binding to a "clamp-motor" protein, like the WASP-complex, with induced polymerization. The resulting local pseudoequilibrium F-actin concentrations are $a^{b}, a^{f}$, and $a^{c}$, respectively. See equations (31) and (33) for a quantitative model.

Finally, also the two hyperbolic equations (7) and (14) require zero-influx conditions, so that for the transported concentrations $m_{b}$ and $c_{a}$ we have to impose zero-Dirichlet conditions only in cases of $V>0$ or $V_{c}>0$, respectively. In the latter case the inward normal velocity of $\mathbf{v}_{c}$ is defined in analogy to (17), noting that under the modeling hypothesis (10) we anyway have $V_{c}=\kappa V$. 


\subsection{Force balance equations}

\subsubsection{Two-phase flow equations for cytoskeleton and cytosol.}

Since cell movement usually occurs on a time scale of minutes, and cell sizes usually are in the order of tens of microns, already the cytosol, with its consistency similar to an aqueous viscous fluid, has low Reynolds number. The more this is true for the cytoskeleton with consistency similar to a dense viscoelastic gel. The viscoelastic properties of the cytoplasm have been experimentally studied [18, 19] and also mathematically modeled [11, 49], whereby the simple model of a Maxwell fluid seems to serve as a quite good description. This means, that elastic properties are mainly effective on a shorter time scale of seconds and dominated by viscous effects on a medium scale of minutes. Moreover, in contrast to a passive elastic material, the cytoplasm mostly stays under active contractile stress as exerted by myosin-II oligomers (in the order of $\mathrm{kPa}$ ), which is able to break weaker cross-links of the F-actin network e.g. by $\alpha$-actinin or filamin, so that the contraction forces are equilibrated only by viscous and frictional forces.

Under these assumptions, the highly viscous two-phase creeping flow model for cytoplasm, originally proposed 25 years ago by Dembo et al. [14 and so far extensively applied, see e.g. 11, 26, can be condensed into a pseudo-stationary linear elliptic system for the mean F-actin velocity $\mathbf{v}$ and the effective hydrostatic pressure $p$ on $\Omega(t)$ :

$$
\begin{aligned}
\nabla \cdot \mu \theta \widetilde{\nabla} \mathbf{v}+\nabla\left(S\left(\theta, m_{b}\right)-p\right) & =\Phi\left(\theta, c_{s a}\right) \mathbf{v}_{c}, \\
\nabla p & =\varphi \theta(\mathbf{v}-\mathbf{w}) .
\end{aligned}
$$

Here the generalized (elliptic) Stokes equation (19) involves the effective stress $S\left(\theta, m_{b}\right)$ as defined in equation 24 and the symmetrized displacement rate $\widetilde{\nabla} \mathbf{v}$ (see e.g. [35]). Moreover, $\mathbf{v}_{c}$ is defined in (11), and $\mu, \Phi$ denote the coefficients of bulk viscosity and substratum friction, see 230 . Because of negligible cytosol viscosity the simple Darcy law (20) suffices as a model description, where the coefficient $\varphi$ measures the internal twophase flow friction. From the last equation one explicitly solves for the cytosol velocity $\mathbf{w}=\mathbf{v}-(1 / \varphi \theta) \nabla p$, so that the total volume flux is

$$
\mathbf{W}=\mathbf{v}+(1-\theta)(\mathbf{w}-\mathbf{v})=\mathbf{v}-\frac{1-\theta}{\varphi \theta} \nabla p .
$$

Insertion into the mass-balance equation (1) then yields the generalized (elliptic) Laplace equation

$$
\nabla \cdot \frac{1-\theta}{\varphi \theta} \nabla p=\nabla \cdot \mathbf{v}
$$

Notice that here the ellipticity degenerates for marginal volume fractions $0<\theta<1$, which can be relevant in cases where $\theta \rightarrow 0$ at boundary points of cytoskeleton disruption, see (18) above. 
We remark that together with the hyperbolic mass balance equation (3) the linear elliptic system 1922 constitutes generalized pseudo-stationary Navier-Stokes equations for the F-actin network as a compressible, highly viscous and reactive fluid. It is mutually coupled to the mass concentrations in equations (7) and (16) via a myosin-mediated contractile stress term appearing in $S\left(\theta, m_{b}\right)$, see eq. (24) below, and an adhesion-mediated friction coefficient

$$
\Phi\left(\theta, c_{s a}\right)=\Phi_{0} c_{s a} \theta
$$

In this way the right hand side of $(19)$ reads $\mathbf{F}_{v}=\Phi \mathbf{v}_{c}=c_{s a} \mathbf{F}_{c}$, with the frictional force $\mathbf{F}_{c}$ per doubly bound integrin defined in $(12)$ above.

Tracing these model equations back to their derivation [12, 2, 35] provides us not only with precise biophysical conditions on $\mathbf{v}$ and $p$ at the moving boundary $\Gamma$ (see next paragraph), but also with genuine nonlinear parameter functions being based on thermodynamical reasoning at the molecular scale: The function $S$ in 19 represents the effective stress in the network phase, which is induced by molecular interactions between the Factin filaments themselves as well as by thermic interactions with solvent molecules in the cytosol. It is generally expressed as the weighted negative sum $S=-\theta P_{a}-(1-\theta) P_{s}$ of corresponding pressures $P_{a}$ and $P_{s}$ which are applied to any network volume element and any cytosol element, respectively. Passive elastic stresses may be neglected since these are already relaxed on the creeping flow timescale. When finding a free binding site on filaments, previously single bound myosin-II tetramers exert power stroke motor forces with their free myosin heads, cf. Figs. 2 and 4 . Thus, an attractive stress $-P_{a}=\psi_{0} m_{b}$ is applied, where the simple coefficient $\psi_{0}$ comprises binding affinity, power stroke probability and the mean applied force per stroke. On the other hand, standard Gibbs free energy arguments suggest a molecular solvent pressure $P_{s}=-\sigma_{0} \ln (1-\theta) /(1-\theta)$, see [2]. Then we arrive at expressions for the

$$
\begin{aligned}
\text { effective stress } & S=S\left(\theta, m_{b}\right) & =\psi\left(\theta, m_{b}\right)-\sigma(\theta) \\
\text { contractile stress } & \psi\left(\theta, m_{b}\right) & =\psi_{0} \theta m_{b} \\
\text { swelling pressure } & \sigma(\theta) & =\sigma_{0}|\ln (1-\theta)|,
\end{aligned}
$$

see Fig. 5 for corresponding plots with $m_{b}=m_{b}^{*}$ in equation (9). We emphasize that the effective stress function in equation (24) sums up the contributions from the cytoskeleton and the cytosol, so that both phases are not separated anymore. However, when deriving the corresponding natural boundary conditions for the elliptic problem, then the two phases have to be considered separately again.

\subsubsection{Stress and pressure balance conditions at the free boundary.}

Recall that according to (2) the cell edge $\Gamma(t)$ always moves together with the normal component of the total cytoplasm flux $\mathbf{W}$ as quantified in (21). Then, from (17) also the 


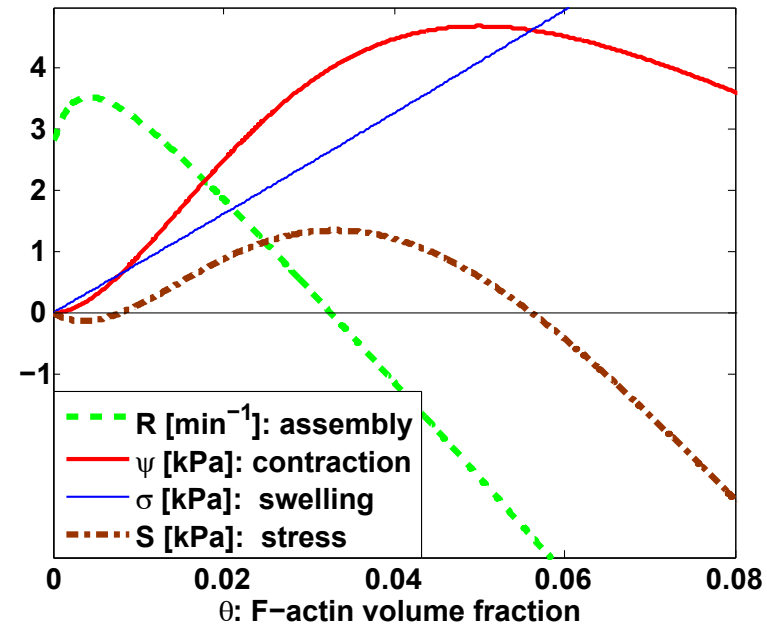

Figure 5: Plots of F-actin model functions. Dashed curves: net assembly rate $R(\theta)$, equation (4), and effective stress $S(\theta)=\psi(\theta)-$ $\sigma(\theta)$, equation (24). Continuous curves: contractile stress $\psi$ defined in equation (41) and swelling pressure $\sigma$, equation (26).

relative inward normal F-actin flux is determined as proportional to the inward normal pressure gradient on $\Gamma(t)$

$$
\theta V=\theta\left(\dot{\Gamma}-\nu_{\Gamma} \cdot \mathbf{v}\right)=-\frac{1-\theta}{\varphi} \nu_{\Gamma} \cdot \nabla p
$$

This explicit linear relation between free boundary speed, normal F-actin velocity and pressure gradient serves as an extra free boundary condition in addition to the set of boundary conditions that are necessary for uniquely solving the linear elliptic system (19) and (22) on the given domain $\Omega(t)$. These depend on the particular model choice of boundary pressure functions at the cell edge membrane, but also on the outcome of the F-actin flow requirement $V \geq 0$ in (17).

Using the general derivations in [2], eqs. (58-62), one obtains separate pressure balance conditions for each of the two phases at all free boundary points of $\Gamma(t)$ satisfying $V>0$,

$$
\begin{aligned}
\text { cytoskeleton } & -\nu_{\Gamma} \cdot \mathbb{T}_{a} \cdot \nu_{\Gamma}+\theta p=\theta P_{a}^{\Gamma} \\
\text { and cytosol } & (1-\theta) p+\sigma(\theta)=(1-\theta) P_{s}^{\Gamma}
\end{aligned}
$$

with the intrinsic stress tensor $\mathbb{T}_{a}=\mu \theta \widetilde{\nabla} \mathbf{v}+\psi\left(\theta, m_{b}\right) \mathbb{I}$. These equations mean that at those parts of the tip plasma membrane, which are exposed to the cytoskeleton network $(\theta)$ or to the cytosol $(1-\theta)$, respectively, the sum of internal pressures is in balance with a certain boundary cytoskeleton pressure $P_{a}^{\Gamma}$ or boundary cytosol pressure $P_{s}^{\Gamma}$ at each volume element. Modeling expressions for these pressures will be given in the following paragraph.

Summing up both pressure balance equations (28) and (29) yields the general Neumanntype condition for $\mathbf{v}$ on $\Gamma(t)$

$$
\nu_{\Gamma} \cdot \mathbb{T}_{a} \cdot \nu_{\Gamma}-(\sigma(\theta)+p)+\theta P_{a}^{\Gamma}+(1-\theta) P_{s}^{\Gamma}=0,
$$


which is now valid without restriction, see [2], eq. (58), i.e. also in boundary points where the condition $V=0$ holds. However, then the Dirichlet-type boundary condition 29 for $p$ has to be replaced by a corresponding inequality, and insertion of $V=0$ into (27) provides a Neumann condition for $p$ instead. In particular we have $\nu_{\Gamma} \cdot \nabla p=0$, which also holds in case of disruption with $V>0$ and $\theta=0$.

Thus, in any constellation of the mentioned conditions on $\Gamma(t)$, for fixed time $t$ and given profile of all protein concentrations, we obtain a well-posed elliptic boundary value problem for $\mathbf{v}$ and $p$ to be solved on $\Omega(t)$ (eventually by numeric iteration and/or conjugate gradient method, cf. [35]). Finally, the normal speed $\dot{\Gamma}$ of the free boundary can be calculated by using equation (27). This is also possible in the case of network disruption at some boundary point $\mathbf{x}_{0} \in \Gamma(t)$ with $\theta\left(t, \mathbf{x}_{0}\right)=0$, since then $V\left(t, \mathbf{x}_{0}\right)>0$ is obtained as the L'Hospital-limit of $-\frac{1-\theta(t, \mathbf{x})}{\theta(t, \mathbf{x})} \nu_{\Gamma} \cdot \nabla p(t, \mathbf{x})$ as $\mathbf{x} \rightarrow \mathbf{x}_{0}$ from the interior.

\subsubsection{Boundary pressure functions at the cell edge.}

We only consider creeping cell migration in a medium that is not under external pressure or stress. Therefore, in a purely physical model, the boundary pressures $P_{a}^{\Gamma}$ and $P_{s}^{\Gamma}$ along the cell edge $\Gamma(t)$ should be set to zero. However, the edge is physically defined as the lamellar tip, where the ventral and dorsal part of the surrounding plasma membrane meet. Thus, if $\tau_{\Gamma}$ denotes the scalar tension value of the dorsal plasma membrane at the cell edge (see the following paragraph) then this tension appears as a boundary tip pressure acting on both phases, the cytoskeleton and the cytosol. Moreover, in addition to the counteracting cytoskeleton and cytosol pressure at the left hand sides of eqs. (28) and (29), there could be extra pressures due to active polymerization forces, which usually are generated at barbed actin filament ends that can become exposed to the tip membrane in two variants, see Fig. 4 above:

- Brownian ratchet model: Assume that at a point of the lamellar tip $\Gamma(t)$ a fraction $a^{B}$ of filaments is bound to membrane proteins in a fast pseudo-steady state equilibrium with the actual F-actin concentration $a=\theta a_{\max }$. Then from the remaining network with concentration $a^{F}(a)=a-a^{B}(a)$, Arp2/3 induced branching can occur. The barbed filament ends are more or less normally exposed to the tip membrane with concentration $a^{f}=a^{f}(a)=\alpha_{0}^{f} \cdot \operatorname{Arp} 0 \cdot \frac{a^{F}}{K_{a}+a^{F}}$ with suitably chosen coefficients, see also (5). Then, due to insertion of G-actin in-between fluctuating filament and membrane there appears a free polymerization pressure

$$
p^{f}=\pi_{0}^{f} \cdot a^{f}(a)
$$

with a ratchet coefficient $\pi_{0}^{f}>0$ depending on the free energy of one monomer addition, see relevant force estimations e.g. in [41].

- Clamp-motor model: Alternatively or additionally, a certain fraction of tip bound actin filaments with concentration $a^{c}=a^{c}(a)<a^{B}(a)$ can be bound to WASP-like 
membrane proteins, which serve as filament-end-tracking motors. By means of an energy consuming polymer elongation process at the clamped end of the filament, these proteins push the bound filament outward, thus leading to a clamp polymerization pressure

$$
p^{c}=\pi_{0}^{c} \cdot a^{c}(a) .
$$

Here again, the clamp-motor coefficient $\pi_{0}^{c}>0$ depends on the energy of one monomer translocation, see [16].

The sum of these active polymerization pressures would induce an averaged relative normal inward mass flux $\theta V \geq 0$ of the whole cytoskeleton, based on the particular inflows of $a^{f}$ and $a^{c}$-filaments satisfying $\theta V=a^{f} V_{f}=a^{c} V_{c}$. However, this flow experiences a viscous resistance from the remaining fixed filaments (concentration $a^{B}-a^{c}$ ), with a viscosity coefficient that could increase in the presence of bound myosin molecules. Thus we get the total tip polymerization pressure

$$
P_{\text {poly }}=p^{f}+p^{c}-\mu_{\Gamma}\left(m_{b}\right)\left(a^{B}-a^{c}\right) a_{\max } V .
$$

Clearly, here the relative inward velocity $V$ is the one defined by the normal component of $\mathbf{v}$ in relation to $\dot{\Gamma}$, see 17 . For a similar resistance model based on elastic cross-linking see [23].

Finally, since this intrinsic boundary pressure $P_{\text {poly }}$ acts onto the cytoskeleton volume fraction, while simultaneously inducing a corresponding counter-pressure onto the cytosol volume fraction, we can state the following distribution for the boundary pressures generated at the membrane

$$
\begin{aligned}
\theta P_{a}^{\Gamma} & =\theta\left(1-\kappa_{\Gamma}\right) \tau_{\Gamma}+P_{\text {poly }}, \\
(1-\theta) P_{s}^{\Gamma} & =\left(1-\theta+\theta \kappa_{\Gamma}\right) \tau_{\Gamma}-P_{\text {poly }},
\end{aligned}
$$

where we introduce a weight factor, $0 \leq \kappa_{\Gamma}<1$, measuring the relative effect of membrane tension $\tau_{\Gamma}$ onto the cytosol phase and possibly depending on the not-explicitly modeled tip geometry. Then, by substituting $p$ from (29) into (30) we obtain the generalized Neumanntype boundary condition for $\mathbf{v}$ in all points of $\Gamma(t)$ where the network is attached, $a^{B}>0$, and where $V>0$ holds:

$$
\nu_{\Gamma} \cdot \mathbb{T}_{a} \cdot \nu_{\Gamma}-\sigma(\theta)-\frac{a_{\max }}{1-\theta} \mu_{\Gamma}\left(m_{b}\right)\left(a^{B}-a^{c}\right) V+\frac{1}{1-\theta}\left(\sigma(\theta)+p^{f}+p^{c}-\theta \kappa_{\Gamma} \tau_{\Gamma}\right)=0 .
$$

This means, that the mean inward F-actin polymerization speed $V \geq 0$ on the moving cell edge $\Gamma(t)$ is implicitly determined by solving the linear elliptic system $(19)$ and $(22)$ for $\mathbf{v}$ and $p$ and satisfying all boundary conditions. Thereby the Neumann condition above contains all membrane protruding pressure terms in series, namely the swelling pressure $\sigma$, the polymerization pressures $p^{f}$ and $p^{c}$ induced by a Brownian ratchet or an clamp-motor mechanism, as well as a counteracting stress due to dorsal membrane tension $\tau_{\Gamma}$. 


\subsubsection{Global force balance at the adhesive substratum.}

There are two kinds of forces exerted by the migrating cell (fragment) onto the fixed flat substratum: the integrin-adhesion mediated active frictional force represented by the vector field $\mathbf{F}_{v}$ on the right hand side of Stokes' equation $(19)$, and an analogous passive frictional force $\mathbf{F}_{u}$ due to motion of the dorsal plasma membrane along the substratum:

$$
\begin{aligned}
& \mathbf{F}_{v}=c_{s a} \mathbf{F}_{c}=\Phi\left(\theta, c_{s a}\right) \mathbf{v}_{c} \\
& \mathbf{F}_{u}=\Phi_{u} \mathbf{u}
\end{aligned}
$$

with $\Phi$ as in 23 and an assumed friction constant $\Phi_{u} \geq 0$. Supposing that on the substratum no other forces are applied than these, then their integral sum has to vanish, so that the zero force balance holds:

$$
0=\int_{\Omega(t)}\left(\mathbf{F}_{v}+\mathbf{F}_{u}\right)
$$

Furthermore, if we assume that the dorsal membrane, viewed as a 2-dimensional incompressible fluid satisfying the zero-divergence condition (10), has relatively low viscosity, then the membrane tension $\tau_{u}$ induced by the frictional flow can be defined according to Darcy's law

$$
\nabla \tau_{u}=\Phi_{u} \mathbf{u}
$$

and thus determined as solution of the Laplace equation $\Delta \tau_{u}=0$ with Neumann boundary condition $\nu_{\Gamma} \cdot \nabla \tau_{u}=0$, see (10). Then the zero force balance (39) together with 190 implies that the boundary tension values $\tau_{\Gamma}=\left.\tau_{u}\right|_{\Gamma(t)}$, which are uniquely determined up to a constant, necessarily fulfill the integrability condition $\int_{\Gamma(t)}\left(\mu \theta \widetilde{\nabla} \mathbf{v}+S\left(\theta, \mu_{b}\right)-p+\tau_{\Gamma}\right) \nu_{\Gamma}=0$, which by insertion of the Neumann boundary condition (30) and by using the symmetry of $\widetilde{\nabla} \mathbf{v}$ reduces to the equivalent necessary condition $\int_{\Gamma(t)}\left(\theta P_{a}^{\Gamma}+(1-\theta) P_{s}^{\Gamma}-\tau_{\Gamma}\right) \nu_{\Gamma}=0$. Indeed, this condition is fulfilled for the boundary pressure model functions that were chosen in the preceding paragraph, (34) and (35), because then even the integrand in the previous condition vanishes.

\section{Results of model simulations}

\subsection{Spontaneous cell polarization in the 2-D model}

We have simulated the adhesive motion of a flat cell or cell fragment represented by a 2-dimensional domain, $\Omega(t)$, with moving cell edge or lamellar tip, $\Gamma(t)=\partial \Omega(t)$, under certain simplifying assumptions:

1. The lower dorsal membrane sticks to the substratum $(\mathbf{u}=0)$ and there exists a small membrane tension $\tau_{\Gamma}>0$, constant over the whole cell edge. 
2. There occurs no active polymerization pressure at the cell edge $\left(p^{f}=p^{c}=0\right)$, only the similar swelling pressure $\sigma(\theta)$ and the hydrostatic pressure $p$ can push the boundary.

3. Disruption of the F-actin network from the lamellar tip $\Gamma(t)$ can locally occur if the network tension exceeds a certain threshold that might depend on the fraction of membrane-bound actin filaments $a^{B}=A_{\frac{a}{K_{B}+a}}$.

4. The free myosin-II concentration is at a fixed constant level $m_{f}^{0}>0$ and the amount of F-actin bound myosin-II oligomers is in a pseudo-steady equilibrium $m_{b}^{*}(a)$ according to (9), so that the contractile stress is only a function of $\theta=a / a_{\max }$ :

$$
\psi(\theta)=\psi_{0} \theta m_{b}^{*}=\frac{\psi_{0} \alpha_{m} m_{f}^{0}}{a_{\max } \delta_{m}^{0}} \frac{\theta^{2}}{1+\theta^{2} / \theta_{\mathrm{opt}}^{2}} .
$$

More details and a list of chosen parameters can be found in [35] (Section 1 and Table 2). Since unpolarized cells and cell fragments, as observed under various conditions [63, 64, attain a quite regular circular shape, we choose as initial condition a circle $\Omega(0)$ of radius $6 \mu \mathrm{m}$. Moreover, in order to mimic the radial spreading of cells after exposure onto a flat substratum, we start with constant integrin densities and radially symmetric initial configuration for the volume fraction $\theta$, with slightly larger values closer to the center. Due to this initial perturbation, the F-actin concentration rapidly condenses into a central region of high $\theta$, surrounded by a lamella-kind region of low $\theta$, see Fig. 6(a,b). Later, also the actin- and surface-bound integrin adhesion proteins $c_{s a}$ concentrate around this center, see Fig. 6(e). Both phenomena are supported by a strong retrograde F-actin flow, which collects actin filaments and actin bound integrins $c_{a}$ in radial direction from almost everywhere in the periphery. Moreover, the hydrodynamic pressure has its maximum within the center region (data not shown), so that its negative outward gradient represents the squeezed flow of cytosol out of the contracting F-actin network.

At the free cell edge with relatively low F-actin concentration, there occurs a meta-stable equilibration between a positive swelling pressure pushing the lamellar tip outwards and a resisting viscous network tension pulling the tip inwards. For some time, see Fig. 6(b) after 5 minutes, local regions of protrusion or retraction can be observed, which point into varying directions along the cell periphery. Notice that these spatiotemporal fluctuations are not due to the tiny stochastic perturbations that are imposed to the F-actin polymerization rate, but represent the emergent chaotic dynamics of the cytoplasm as a reactive and contractile two-phase fluid [11, 3]. Furthermore, behind a cyclically protruding and retracting free edge we can observe a layer with slightly increased concentration of substrate-and-actin bound integrin $c_{s a}$, see Fig. 6(e). This is the onset of polarization: fresh free integrin proteins are appearing at the protruding part of the edge from the upper membrane, thus also increasing $c_{s a}$ in this region. As a consequence, a larger frictional force of the retrograde $\mathrm{F}$-actin is generated in that direction, inducing a bias into the force 

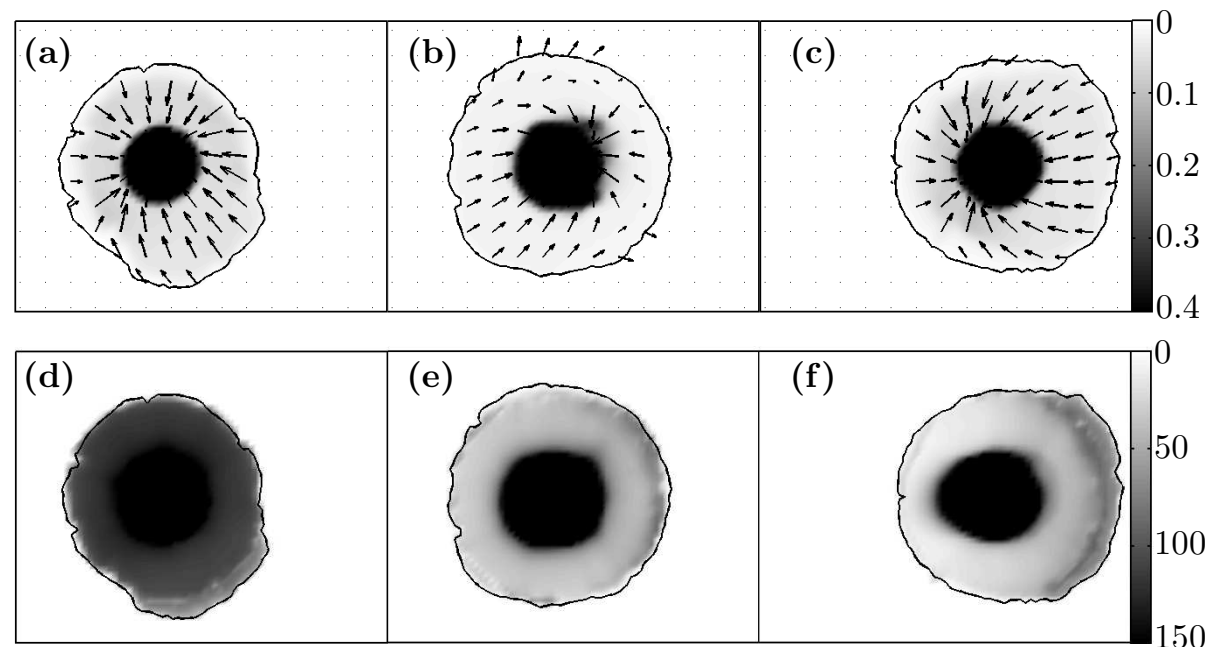

Figure 6: 2-D simulation of a spontaneously polarizing cell (fragment) starting with radially symmetric initial conditions: Spatial distributions shown as pseudocolor plots of F-actin volume fraction $\theta$ in $(a, b, c)$, and concentration of actin-and-surface bound integrin proteins $c_{s a}$ in (d,e,f) at three different time instants: (a,d) 1 min, (b,e) 5 min, and (c,f) 10 min after initialization. The width of the shown region is $22 \mu \mathrm{m}$. 
vector field transduced to the substratum. Thus, the whole cell fragment starts to move in this direction, which later becomes the leading edge of the migrating cell fragment, see Fig. 6(c,f). The emerging polarization of the cell is most clearly expressed in the $c_{s a}$ distribution of Fig. 6(f), where an increasingly dense band is formed behind the leading edge and the central region of focal adhesions is slowly shifted rearwards with increasing migration speed, while becoming deformed in shape similarly to the whole cell fragment.

This simulation is an example for the autonomous formation of a meta-stable unpolarized, almost circular state and its spontaneous transition into a polarized, migrating state of a flat cell fragment. By changing some of the model parameters we can influence the degree of this symmetry-breaking instability, but so far we were not able to reproduce the observed longer-time stability of circular cell fragments, see again [63]. One reason for this failure seems to be, that in our model simplifications we assumed a constant distribution of freely diffusing myosin-II oligomers, contradicting experimental results on clearly expressed gradients of myosin-II concentration decaying towards the cell edge, see e.g. 60]. Therefore we have started to investigate the full coupled model system by including active tip polymerization and the kinetics for myosin-II diffusion, binding and transport, in addition to the already implemented analogous kinetics and dynamics of integrin adhesion molecules. The next section presents the so far achieved results in the most simple but nevertheless quite instructive 1-dimensional situation.

\subsection{Induced onset of cell polarization and migration in the 1-D model}

We have simulated adhesion, polarization and migration of an idealized flat cell fragment having a fixed extension and only one degree of freedom to move, as can be observed experimentally, for example in Fig. 1(c). Thus, in a 1-D cross-section along its moving direction, the fragment is represented by an interval $\Omega(t)=\left[x_{b}(t), x_{b}(t)+L\right]$ of fixed length $L$, moving with body speed $v_{b}(t)=\dot{x_{b}}(t)$, so that the whole kinetics and dynamics within cytoplasm and dorsal membrane can be described by the corresponding 1-dimensional equations and conditions as in Section 3.2 , but now written in cell-centric coordinates, for example $\tilde{\theta}(t, y)=\theta\left(t, x_{b}(t)+y\right)$ or $\tilde{v}(t, y)=v\left(t, x_{b}(t)+y\right)-v_{b}(t)$. Then, assuming the following particular restrictions, we have solved a simplified system of boundary value problems:

1. The actin network is always sticky at the lamellar tips (no disruption), so that $\tilde{\theta}>0$ on the whole closed interval $[0, L]$.

2. We assume active tip polymerization with a simultaneous parallel effect of the brownian ratchet $\left(\pi_{0}^{f}=5 \mathrm{~Pa} / \mu \mathrm{M}\right)$ and the clamp-motor mechanism $\left(\pi_{0}^{c}=5 \mathrm{~Pa} / \mu \mathrm{M}\right)$. Moreover, we suppose that the membrane-bound cortex shear viscosity strongly increases if myosin-II oligomers are bound: $\mu_{\Gamma}\left(m_{b}\right)=0.1\left(1+45 m_{b}\right) \mathrm{Pa} \min / \mu \mathrm{m}$. For the actinbinding membrane proteins at the tip with maximal concentration $A=50 \mu \mathrm{M}$ and 

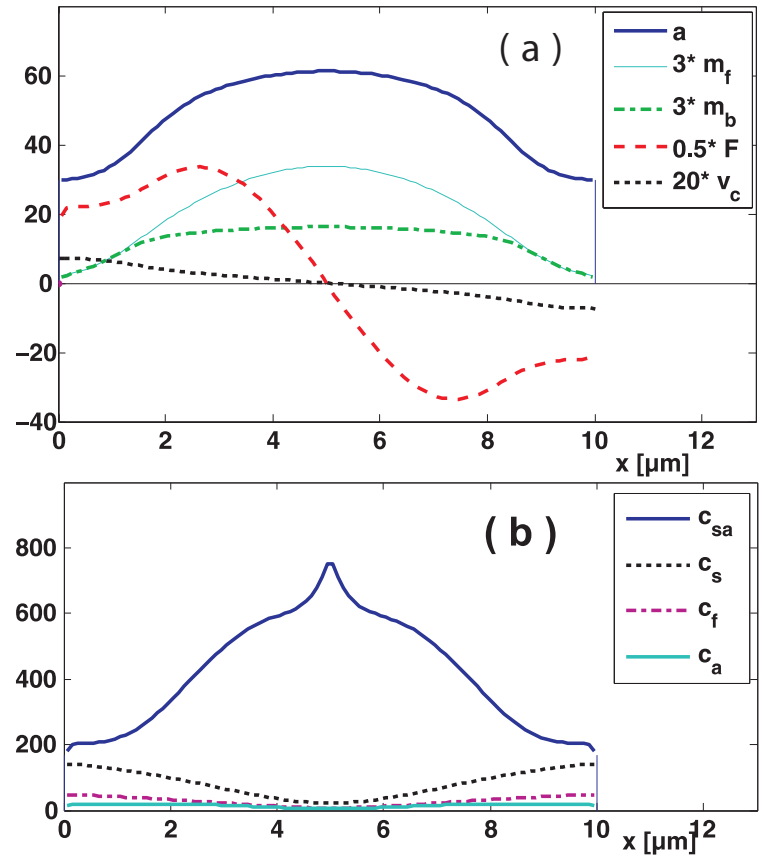

Figure 7: Symmetric unpolarized state of a model cell (fragment) with length $10 \mu \mathrm{m}$ showing the concentration profiles of

(a) F-actin $a$, free and bound myosin-II $m_{f}$ and $m_{b}$, the force $F$ onto the substrate and the effective cortical F-actin velocity $v_{c}$

(b) of integrin proteins in the four different states, namely substrate- and actin-bound $c_{s a}$, substrate-bound $c_{s}$, freely diffusing $c_{f}$, and actin-bound $c_{a}$.

self-enhanced binding with dissociation constant $K=158.1 \mu \mathrm{M}$ we use the pseudoequilibrium $2 a^{B}(a)=A-\sqrt{(A-a)^{2}+2(A+a) K^{2} / a+\left(K^{2} / a\right)^{2}}+K^{2} / a+a$ and $a^{c}=0.1 a^{B}$.

3. The dorsal membrane moves together with the cell (no slip at the tips), so that $\tilde{u} \equiv 0$ and $v_{c}=v=\tilde{v}+v_{b}$. The tip membrane tension difference is $\left[\tau_{\Gamma}\right]_{0}^{L}=\Phi_{u} L v_{b}$ with the minimum always equal to a fixed positive constant $\tau_{0}=25 \mathrm{~Pa}$. Finally, the substrate force balance $(39)$ reads

$$
\Phi_{u} L v_{b}=\int_{0}^{L} \Phi_{0} \widetilde{c_{s a}} \tilde{\theta}\left(\tilde{v}+v_{b}\right)
$$

This is an implicit equation to be solved for the migration speed $v_{b}$, since the Neumann boundary conditions and the right hand side of the elliptic equation (19) ) for $\tilde{v}$ (after eliminating the pressure $\tilde{p}$ ) contain expressions that depend (linearly) on $v_{b}$.

Starting with the same unpolarized initial condition as in the previous Section 4.1, we obtain a similar 1-dimensional symmetric configuration with no cell translocation and a central F-actin plateau, a central maximum of FA (focal adhesion) sites, i.e. $c_{s a}$-integrins, as well as a centripetal F-actin flow, see Fig. 7. In addition, also the concentration of total myosin-II is enriched in the central region, with actin-bound myosin forming a central plateau, consistent with fluorescence pictures of unpolarized keratinocyte fragments, see 
[63. Moreover, the F-actin flow is highest at the boundary (with values $V \sim 0.5 \mu \mathrm{m} / \mathrm{min}$ ) due to the assumed active tip polymerization.

In contrast to the previous 2-D model simulations, here we find robust parameter constellations yielding stability of this symmetric unpolarized state: even quite strong but still subthreshold perturbations of the F-actin concentration at one side induce only transient locomotion together with shifts in most concentration profiles: after some delayed overshooting, the cell fragment returns to its non-moving non-polarized stable state in Fig. 7 . However, if F-actin polymerization is continuously stimulated at one side (mimicking the effect of a chemo- or haptotactic gradient) then, not surprisingly, the cell fragment slowly polarizes and starts to persistently translocate in this direction (data not shown).

Similarly, in order to mimic the mechanical stimulation experiments with keratinocyte fragments as performed in 63, we locally increase the activation (and F-actin-binding) of myosin-II at the left hand side for a certain time (up to $30 \mathrm{sec}$ ), which is thought to be analogous to the experimental push by a micropipette flow pulse, since by local compression of the cytoskeleton, filament alignment and thus myosin action will be enhanced. If time span or amplitude of this myosin-pulse stays subthreshold, the model cell responses only by a transient migration as described above and asymptotically returns to its stable resting state, see the speed curve in Fig. 8(a). However, if the pulse strength exceeds a certain
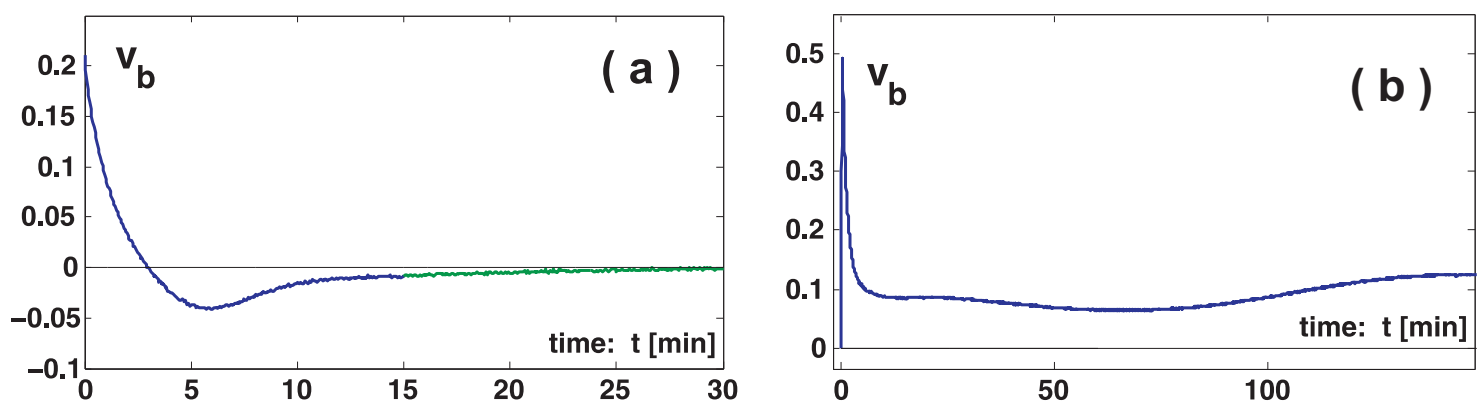

Figure 8: Plots of cell migration velocity $v_{b}$ over time after a perturbation of the unpolarized state in Fig. 7 by imposing an additional activation rate $\alpha_{m}^{+}$for actin-bound myosin-II oligomers over an induction period of $0.5 \mathrm{~min}$ locally at the left cell side: (a) with rate $\alpha_{m}^{+}=20 / \mathrm{min}$, showing a stable convergence of $v_{b}$ towards the unpolarized speed zero, and (b) with rate $\alpha_{m}^{+}=50 / \mathrm{min}$, showing the convergence towards a positive polarized speed $\sim 0.012 \mu \mathrm{m} / \mathrm{min}$.

threshold, myosin-II and F-actin is condensed at the rear, whence active tip polymerization $(V)$ is drastically reduced at this side, so that the cell rapidly starts to migrate in the other direction, see Fig. 8(b). After cessation of the pulse, the migration speed is reduced, but the cell maintains its polarized locomotion state. This induced polarization, as a non-linear threshold behavior, is supported by a further positive feedback mechanism: Due to cell translocation, the focal adhesion sites $\left(c_{s a}\right)$ are successively shifted rearwards relative to 
the cell, which stabilizes the asymmetric polarization and later increases the locomotion speed to a constant asymptotic value $\left(v_{b} \sim 0.12 \mu \mathrm{m} / \mathrm{min}\right)$.
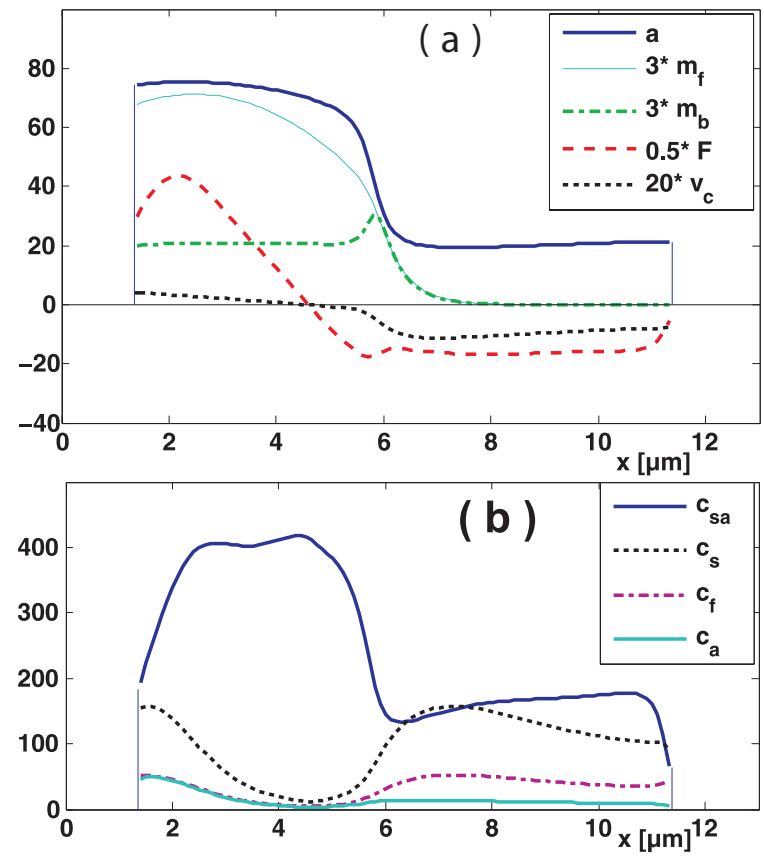

Figure 9: Migrating polarized state showing stable concentration profiles (a) of F-actin, $a$, free and bound myosin-II, $m_{f}$ and $m_{b}$, the transduced locomotion force $F$ and the effective cortical F-Actin velocity $v_{c}$;

(b) of integrin-concentrations as in Fig. 7. Only FA integrins in the $s a$ state are able to transduce force from the contractile actin network to the substrate.

The migration speed is $v_{b}=$ $0.125 \mu \mathrm{m} / \mathrm{min}$.

In this migration state the cell (fragment) attains characteristic concentration profiles, see Fig. 9. Besides the already mentioned polar gradients of F-actin and myosin-II, the most impressive distribution is that of the FA sites: the $c_{s a}$ profile shows a characteristic broad peak behind the leading edge (as in the 2-D case above), followed by a slight decrease and a second plateau of even more condensed adhesion sites in the back part of the cell (fragment) which, however, rapidly decays at the very rear, see Fig. 9(b). This last phenomenon is the theoretically expected and experimentally observed rear release of adhesion sites or integrins, not induced by any directed regulatory protein, but only by the fact that the rear part experiences a steep increase of the force $F$ transduced to the substratum, see the plot in Fig. 9(a). By (37) this is proportional to the F-actin mass flow $\theta v_{c}$ with respect to the substratum: While in the major front part of the migrating cell (fragment) the F-actin flow is retrograde and the centripetally pulling negative force is modest in amplitude, near the trailing edge the direction of flow reverses and the positive force becomes very strong, now centripetally pulling off the focal adhesion sites. Thus, the reason for cell translocation is not that there is "more adhesion" or "stronger force" at the front compared to the rear, as it is asserted in some models, cf. [17. Indeed, for vanishing passive friction $\Phi_{u}$ due to 42 the total force integral even vanishes. The true physical reason for cell migration rather is the aforementioned asymmetry in the polarized cell state, expressed by a wide front region with modest rearward force and short rear region with strong forward force. 
We finally investigate the dependence of migration speed on two cell physiologically important parameters, namely the adhesiveness of the substratum quantified by the relative number of available adhesion sites Adh0, and the responsiveness of the F-actin network measured, for instance, by the concentration Arp0 of activated Arp2/3 proteins. These are mainly responsible for controlling F-actin polymerization by available free filament ends as they appear in the net assembly rate (4) and the ratchet polymerization pressure (31).
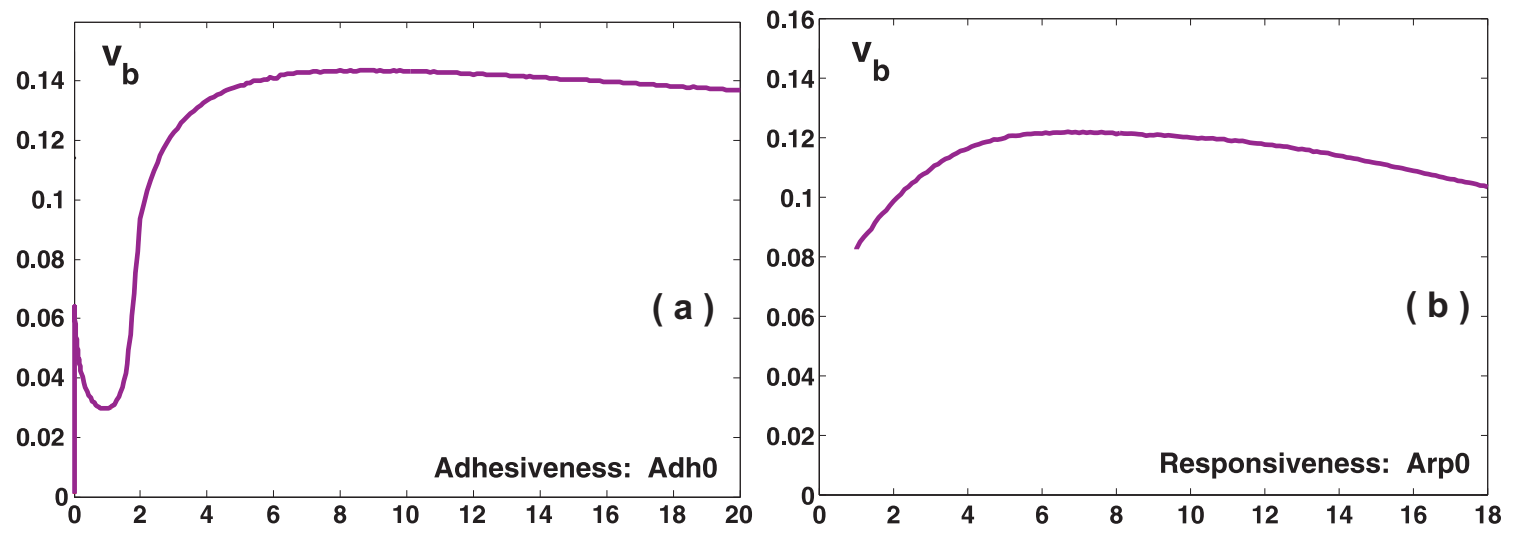

Figure 10: Migration speed $v_{b}$ of a model cell in its stable polarized state, plotted over varied parameters (a) of adhesiveness Adh0, relative amount of adhesion binding sites on the substratum (e.g. fibronectin coating) (b) of F-actin responsiveness expressed by $\operatorname{Arp} 0[\mu \mathrm{M}]$, the cytoplasmic concentration of activated Arp2/3 complexes.

The results depicted in Fig. 10 reveal the existence of optimal ranges for both parameters, consistent with earlier modeling results, see [17, 24, and with experimental observations, see e.g. [27, 52].

Particularly, the migration response curve of Fig. 10(a) has been performed for fixed parameter $\operatorname{Arp} 0=10 \mu \mathrm{M}$ and for adhesion values $0 \leq$ Adh $0 \leq 20$, where according to Table 2 the adhesiveness proportionally influences not only the two adhesion rates $\alpha^{+}=$ $\gamma^{+}=\operatorname{Adh} 0 \cdot \alpha$ but also the passive membrane friction coefficient $\Phi_{u}=\operatorname{Adh} 0 \cdot 6 \mathrm{~Pa} \cdot \mathrm{min} \cdot \mu \mathrm{m}^{-2}$. This is based on the idealizing assumption that there are no other relevant exterior forces that would resist locomotion relative to the substratum, than those due to friction of the dorsal membrane with respect to an adhesive coating of e.g. fibronectin or collagen.

Under these hypotheses and parameter choices our model simulations predict a minimal migration speed of $v_{b} \sim 0.035 \mu \mathrm{m} / \mathrm{min}$ for Adh0 $\sim 1$, which increases towards the doubled speed when lowering the adhesiveness to almost zero. This surprising phenomenon is consistent with own experimental measurements of human keratinocyte polarization and migration [37] revealing slightly increased motility of polarized cells on glass compared to those on a low density fibronectin coat. The reason for this can be seen from the 
corresponding profiles of FA concentration $\left(c_{s a}\right)$ and substrate force distribution $(F)$ for the two adhesiveness values Adh0 $=0.01,0.2$, see Fig. 11(a,b). Due to ongoing retrograde
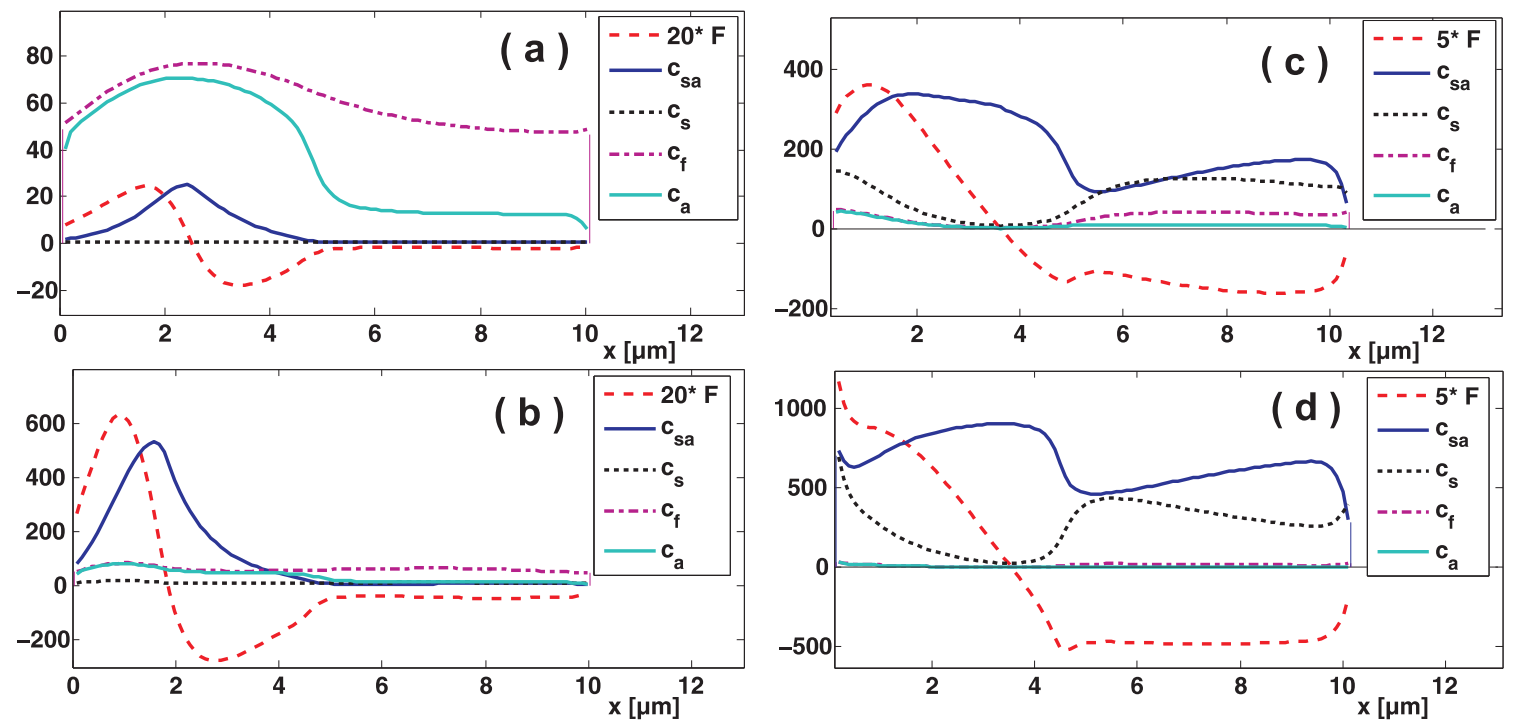

Figure 11: Substrate force distribution $F$ and integrin concentrations in the four different states, plotted for varying adhesiveness parameter Adh0 with corresponding migration speed $v_{b}$ as in Fig. 10(a): (a) 0.01, speed $0.06 \mu \mathrm{m} / \mathrm{min}$, (b) 0.2, speed $0.04 \mu \mathrm{m} / \mathrm{min}$, (c) 3.0 , speed $0.125 \mu \mathrm{m} / \mathrm{min}$, and (d) 20.0 , speed $0.138 \mu \mathrm{m} / \mathrm{min}$.

flow of F-actin, working against internal viscosity and drag, the polarized cell (fragment) gathers the few FA sites on the back side in a way that the local FA and force distributions are almost symmetric (for almost vanishing adhesiveness Adh0 $=0.01$ ) but still with an additional negative (pulling) force plateau on the front side (though of tiny absolute value $|F| \sim 0.05 \mathrm{~Pa}$ ). For increased but still small adhesiveness (Adh0 $=0.2$ ), the much more frequent FA sites start to accumulate at the rear, thus the asymmetry of forces is enhanced and the increased friction reduces the migration speed.

On the other hand, for further increasing adhesiveness, Adh0 $>1$, the front plateau of pulling forces at the enriched FA 'carpet' is proportionally increased, see Fig. 11. (c,d). However, the dominant reason for the non-linear speeding-up response is the prominent increase of disruptive forces $\mid F_{c}$ (rear) $\mid$ up to values of Adh0 $\sim 2$, leading to a drastic reduction of resistive FA sites at the very rear, see the corresponding indicator curves in Fig. 12(a). Only for very large adhesiveness, Adh0 $\sim 20$, the FA sites again start to accumulate at the very rear in spite of a strong disruptive force there, as can be seen in Fig. 11(d), causing a slight decrease in migration speed. Thus, while an adhesiveness between 5 and 12 leads to a saturated optimality in migration speed, within the range $1<$ Adh0 $<4$ the cell (fragment) has a high sensitivity for responding to an increase of 

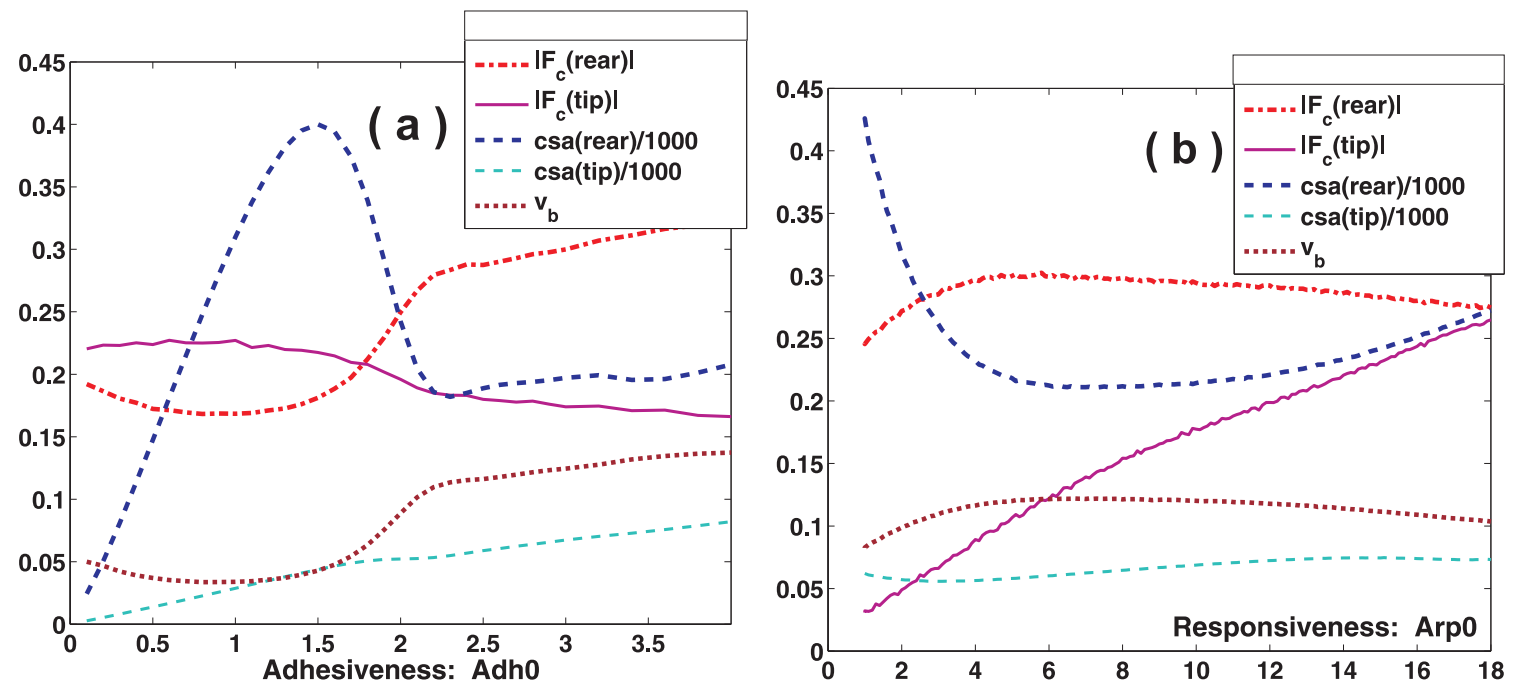

Figure 12: Variation of migration speed $v_{b}$ and certain indicator functions at the cell edge in dependence of (a) adhesiveness Adh0 and (b) F-actin responsiveness Arp0 $[\mu \mathrm{M}]$ : Plotted are the load $\left|F_{c}\right|$ per FA site at the cell rear (bold) and tip (light), and the FA concentration $c_{s a}$ at the rear (bold, dashed) and tip (light, dashed). Ripples in the force curves reflect the slight stochastic noise that was added in the force equation 19 . 
adhesiveness with an up to 4-fold increase of speed. Carried over to the mean forward speed of competing leading lamellae in a whole cell model (as for the 2-D simulations in Fig. 6), this could be used to explain polarization and haptotaxis of cells in spatial adhesion gradients, cf. [20].

In order to understand the analogous optimal migration performance for responsiveness parameters in the range between $4 \mu \mathrm{M}$ and $10 \mu \mathrm{M}$ of Arp2 $/ 3$ concentration Arp0, see Fig. 10(b), we plot the same indicator curves for FA concentrations and substrate forces in Fig. 12(b). Again, with increasing Arp2/3-induced actin polymerization there is an almost linear increase in pulling force at the tip (due to increased polymerization velocity $V$ there) while the concave migration speed curve directly corresponds to the proportional curve for the disruptive forces at the rear and the resulting convex curve for the accumulated FA sites there. For larger Arp0 values the growing accumulation of FA sites again leads to a slight speed reduction. Finally, the about $50 \%$ gain of speed for increasing responsiveness in the range $1 \mu \mathrm{M}<\operatorname{Arp} 0<4 \mu \mathrm{M}$ could again be carried over to a competing lamellar protrusion response in spatial chemotactic gradients, which are known to stimulate F-actin polymerization at the leading front.

\subsection{Migration speed in a simplified 1-D model}

In order to explore, which properties of our coupled cytoplasm-adhesion model are essential for obtaining the 1-D simulation results in the preceding section, we simplified the model by freezing the following variables and parameters, cf. [45]:

1. Assuming fast diffusion and F-actin binding of free myosin-II oligomers, we take the pseudo-steady state condition (9) for bound myosin-II, but with the equivalent dissociation rate $\delta_{m}(a)=\delta_{m}^{0} \exp \left(-2 a / a_{\text {opt }}\right)$ instead of $(8)$, and obtain as contractility $\psi(\theta)=\widetilde{\psi_{0}} \cdot \theta^{2} \exp \left(-2 \theta / \theta_{\text {opt }}\right)$ with a coefficient $\widetilde{\psi_{0}}$ analogous to 41 .

2. Except the FA friction function $\Phi$ we set all other friction coefficients $\left(\varphi, \Phi_{u}\right)$ to zero. Thus, the cell migration speed is determined by the zero integral in 42 .

3. All 'exterior' pressures or tensions at the free membrane tip $\left(\pi_{0}^{f}, \pi_{0}^{c}, \tau_{\Gamma}\right)$ are assumed to vanish, in particular, there occurs no active polymerization $(V \equiv 0)$.

Starting with uniform FA concentration and a minor asymmetry in the distribution of actin, the system reaches a polarized and migrating state and the distributions of the various concentrations exhibit the same characteristics as in the full model (Fig 9). We again analyze the effect of substratum adhesiveness and F-actin responsiveness on the migration speed, see Fig. 13. In contrast to the observations for the full model (see Fig. 10), a saturation behavior of the migration velocity emerges with increasing adhesiveness instead of an optimal range. However, the responsiveness of the F-actin-network has an optimal value as in the full model. 


\begin{tabular}{lll}
$\mathbf{S y m b o l}$ & Meaning & Units \\
\hline$\theta(t, x)$ & Volume fraction of F-actin & dimensionless \\
$a(t, x)$ & Concentration of F-actin & $\mu \mathrm{M}$ \\
$m_{f}(t, x)$ & Concentration of free myosin-II & $\mu \mathrm{M}$ \\
$m_{b}(t, x)$ & Concentration of F-actin bound myosin-II & $\mu \mathrm{M}$ \\
$c_{f}(t, x)$ & Concentration of free integrin & $\mu \mathrm{m}^{-2}$ \\
$c_{s}(t, x)$ & Concentration of substrate bound integrin & $\mu \mathrm{m}^{-2}$ \\
$c_{a}(t, x)$ & Concentration of actin bound integrin & $\mu \mathrm{m}^{-2}$ \\
$c_{s a}(t, x)$ & Concentration of substrate-and-actin bound integrin & $\mu \mathrm{m}^{-2}$ \\
$R(\theta)$ & F-actin net polymerization rate & $\mathrm{min}^{-1}$ \\
$\psi\left(\theta, m_{b}\right)$ & Contractile stress & $\mathrm{Pa}$ \\
$\sigma(\theta)$ & Swelling pressure & $\mathrm{Pa}$ \\
$S\left(\theta, m_{b}\right)$ & Effective cytoplasmic stress & $\mathrm{Pa}$ \\
$p(t, x)$ & Effective two-phase flow pressure & $\mathrm{Pa}$ \\
$\tau_{u}(t, x)$ & Dorsal membrane tension & $\mathrm{Pa}$ \\
$\mathbf{v}(t, x)$ & Mean F-actin velocity & $\mu \mathrm{m} / \mathrm{min}$ \\
$\mathbf{v}_{c}(t, x)$ & Cortical F-actin velocity & $\mu \mathrm{m} / \mathrm{min}$ \\
$\mathbf{u}(t, x)$ & Dorsal membrane velocity & $\mu \mathrm{m} / \mathrm{min}$ \\
$v_{b}(t)$ & Migration velocity of cell (fragment) body $(1-\mathrm{D})$ & $\mu \mathrm{m} / \mathrm{min}$ \\
$\Phi\left(\theta, c_{s a}\right)$ & Adhesional friction & $\mathrm{Pa} \cdot \mathrm{min} / \mu \mathrm{m}^{2}$ \\
$\mathbf{F}_{v}$ & Active frictional force & $\mathrm{Pa} / \mu \mathrm{m}$ \\
$\mathbf{F}_{c}$ & Local frictional force per adhesion site & $\mathrm{Pa} \cdot \mu \mathrm{m}$ \\
$\mathbf{F}_{u}$ & Passive frictional force at dorsal membrane & $\mathrm{Pa} / \mu \mathrm{m}$ \\
$\Gamma$ & Normal speed of the free boundary $\Gamma(t)$ & $\mu \mathrm{m} / \mathrm{min}$ \\
$V$ & Relative inward F-actin velocity at boundary & $\mu \mathrm{m} / \mathrm{min}$ \\
$P_{a}^{\Gamma}$ & Boundary cytoskeleton pressure & $\mathrm{Pa}$ \\
$P_{s}^{\Gamma}$ & Boundary cytosol pressure & $\mathrm{Pa}$ \\
$\tau_{\Gamma}$ & Membrane tension at boundary (lamellar tips) & $\mathrm{Pa}$ \\
$a^{B}(a)$ & Concentration of tip-bound F-actin & $\mu \mathrm{M}$ \\
$a^{c}(a)$ & Concentration of F-actin bound to clamp-motor proteins & $\mu \mathrm{M}$ \\
$a^{f}(a)$ & Concentration of free filaments exposed to the tip & $\mu \mathrm{M}$ \\
$\mu_{\Gamma}\left(m_{b}\right)$ & F-actin shear viscosity relative to $a^{B}$ filaments & $\mathrm{Pa} \cdot \mathrm{min} / \mu \mathrm{m}$ \\
$p^{f}$ & Free polymerization pressure at tip membrane & $\mathrm{Pa}$ \\
$p^{c}$ & Clamp-motor polymerization pressure & $\mathrm{Pa}$ \\
\hline & & \\
& & \\
\hline
\end{tabular}

Table 1: List of model variables and functions 

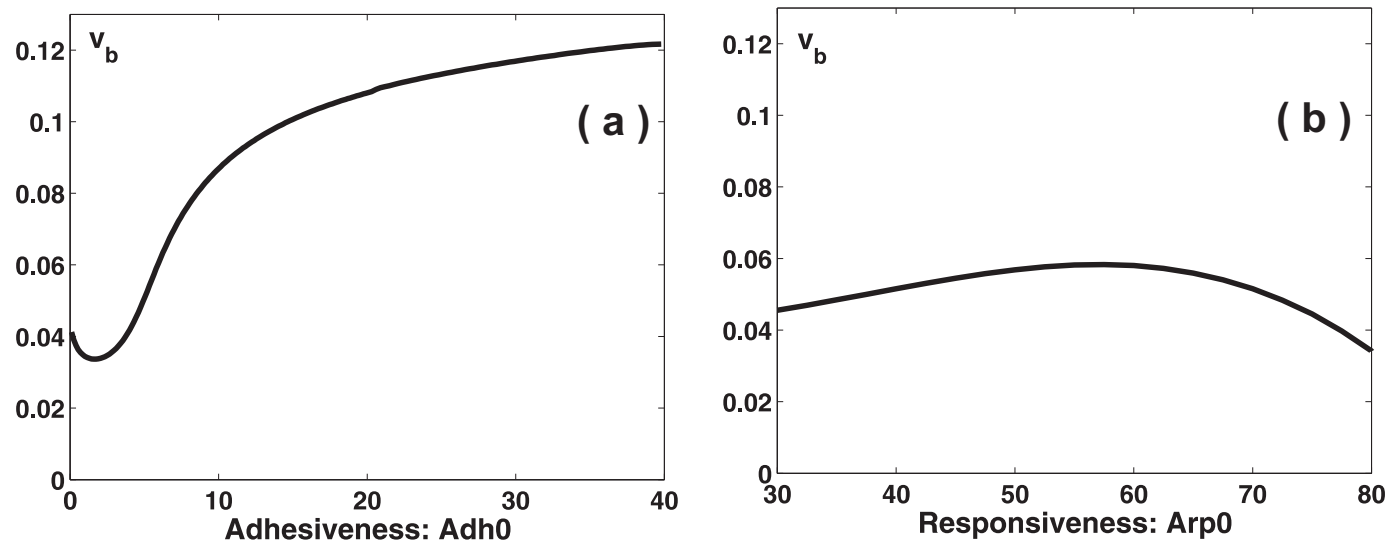

Figure 13: Migration speed $v_{b}$ for a simplified model cell in its stable polarized state in dependence of $(a)$ adhesiveness or $(b)$ F-actin responsiveness as described in Fig. 10.

Further simulations after successive variation of the frozen parameters (see above) reveal, that an optimal range of adhesiveness could be gained only if a passive frictional load $\Phi_{u}>0$ was chosen to increase monotonically with adhesion strength Adh0, as it was assumed in the full model, see Table 2 and Fig. 10(a). Though such a friction appears to be physically realistic, the question remains whether such an optimal velocity response is of general biological relevance. For, the response of living systems is typically guided by adaptation: Migrating cells show the capability to dissolve their focal adhesions by proteolytic cleavage of integrins [55] which could be an adaptive strategy to optimize cellular adhesiveness on various substrates. On the other hand, the adhesiveness could be raised by active segregation of extracellular matrix proteins.

Nevertheless, the universal optimality curve in dependence of F-actin responsiveness, seen in both Figs. 10(b) and 13(b), seems to hold for any mechanical model, which implements a saturating F-actin polymerization by association of regulating proteins as Arp2/3. This indicates that an optimal control of migration velocity by tuning certain chemical processes as F-actin polymerization, is more easy to be realized than tuning adhesion, and thus may have evolved as a generally effective strategy to regulate such mechanical processes as cell adhesion and locomotion.

\section{Discussion and outlook to further modeling}

Based on a larger set of interwoven mass and force balance equations for mechano-chemical processes within the cytoplasm and the surrounding plasma membrane, the presented continuum model is a simplified though already sufficiently complex version of a more com- 


\begin{tabular}{|c|c|c|c|}
\hline Symbol & Meaning & Value & Units \\
\hline$a_{g}$ & Concentration of G-actin & 30 & $\mu \mathrm{M}$ \\
\hline$a_{\max }$ & Maximal concentration of F-actin & 800 & $\mu \mathrm{M}$ \\
\hline$k_{\mathrm{on}}$ & F-actin polymerization rate at plus ends & 696 & $(\min \cdot \mu \mathrm{M})^{-1}$ \\
\hline$k_{\mathrm{off}}$ & F-actin depolymerization rate at plus ends & 258 & $\min ^{-1}$ \\
\hline$\omega$ & F-actin capping rate & 1250 & $\min ^{-1}$ \\
\hline$r$ & F-actin disassembly rate & 0.2 & $\min ^{-1}$ \\
\hline$\varepsilon$ & Basal F-actin nucleation rate & 0.75 & $\min ^{-1}$ \\
\hline Arp0 & Concentration of activated Arp2/3 complexes & 10 & $\mu \mathrm{M}$ \\
\hline$\nu_{a}$ & Arp2/3-induced nucleation rate & 60 & $(\min \cdot \mu \mathrm{M})^{-1}$ \\
\hline$K_{a}$ & Half-saturation concentration for nucleation & 3 & $\mu \mathrm{M}$ \\
\hline$m_{f}^{0}$ & Equilibrium reservoir concentration of free myosin-II & 10 & $\mu \mathrm{M}$ \\
\hline$D_{m}$ & Diffusion coefficient for free myosin-II & 0.5 & $\mu \mathrm{m}^{2} \cdot \min ^{-1}$ \\
\hline$\alpha_{m}$ & F-actin binding rate of myosin-II & 0.5 & $(\min \cdot \mu \mathrm{M})^{-1}$ \\
\hline$\delta_{m}^{0}$ & Dissociation rate of bound myosin-II & 3 & $\min ^{-1}$ \\
\hline$a_{o p t}$ & Optimal F-actin concentration for myosin-II binding & 40 & $\mu \mathrm{M}$ \\
\hline$c_{f}^{0}$ & Reservoir concentration of free integrin at lamellar tips & 50 & $\mu \mathrm{m}^{-2}$ \\
\hline$D_{f}$ & Diffusion coefficient for free integrin & 0.5 & $\mu \mathrm{m}^{2} \cdot \min ^{-1}$ \\
\hline Adh0 & Number of available substrate sites per integrin & 3 & dimensionless \\
\hline Talin & F-actin association factor & 0.0125 & dimensionless \\
\hline Fifactor & Focal complex factor (intracellular) & 50 & dimensionless \\
\hline Fefactor & Focal adhesion factor (extracellular) & 50 & dimensionless \\
\hline$\alpha^{-}$ & Dissociation rate for substrate bound integrin & $\alpha=5$ & $\min ^{-1}$ \\
\hline$\alpha^{+}$ & Free integrin binding rate to substrate & $\operatorname{Adh} 0 \cdot \alpha$ & $\min ^{-1}$ \\
\hline$\beta_{0}^{+}$ & F-actin binding rate for substrate bound integrin & $\alpha \cdot$ Talin & $(\min \cdot \mu \mathrm{M})^{-1}$ \\
\hline$\beta_{0}^{-}$ & FA dissociation of the F-actin link & $\alpha /$ Fifactor & $\min ^{-1}$ \\
\hline$\delta_{0}^{+}$ & Free integrin binding rate to $\mathrm{F}$-actin & $\alpha \cdot$ Talin & $(\min \cdot \mu \mathrm{M})^{-1}$ \\
\hline$\delta^{-}$ & Dissociation rate for $\mathrm{F}$-actin bound integrin & $\alpha$ & $\min ^{-1}$ \\
\hline$\gamma^{+}$ & Substrate binding rate for F-actin bound integrin & $\operatorname{Adh} 0 \cdot \alpha$ & $\min ^{-1}$ \\
\hline$\gamma_{0}^{-}$ & FA dissociation rate of the substrate link & $\alpha /$ Fefactor & $\min ^{-1}$ \\
\hline$\rho_{\beta}$ & Exponential FA-rupture coefficient for F-actin link & 11.7 & $(\mathrm{~Pa} \cdot \mu \mathrm{m})^{-1}$ \\
\hline$\rho_{\gamma}$ & Exponential FA-rupture coefficient for substrate link & 11.7 & $(\mathrm{~Pa} \cdot \mu \mathrm{m})^{-1}$ \\
\hline$A$ & Total free $\mathrm{F}$-actin binding sites on boundary $\Gamma(t)$ & 50 & $\mu \mathrm{M}$ \\
\hline$\pi_{0}^{f}$ & Ratchet polymerization pressure coefficient & 0.0125 & $\mathrm{~Pa} \cdot \mu \mathrm{M}^{-1}$ \\
\hline$\pi_{0}^{c}$ & Clamp-motor polymerization pressure coefficient & 0.0125 & $\mathrm{~Pa} \cdot \mu \mathrm{M}^{-1}$ \\
\hline$\kappa_{\Gamma}$ & Tip curvature weight factor for membrane tension & 0.5 & dimensionless \\
\hline$\alpha_{0}^{f}$ & Relative amount of exposed barbed ends per Arp $2 / 3$ & 2 & dimensionless \\
\hline$\psi_{0}$ & Contractile stress per bound myosin-II & 0.0163 & $\mathrm{~Pa} \cdot \mu \mathrm{M}^{-1}$ \\
\hline$\widetilde{\psi}_{0}$ & Strength of contractile stress (simplified model) & 0.625 & $\mathrm{~Pa}$ \\
\hline$\sigma_{0}$ & Strength of the swelling pressure & 0.125 & $\mathrm{~Pa}$ \\
\hline$\mu$ & Viscosity of the F-actin network phase & 0.625 & $\mathrm{~Pa} \cdot \min$ \\
\hline$\kappa$ & Cortical slip parameter for the F-actin flow & 0.5 & dimensionless \\
\hline$\varphi$ & Drag coefficient between network and solvent & 2 & $\mathrm{~Pa} \cdot \min \cdot \mu \mathrm{m}^{-2}$ \\
\hline$\Phi_{0}$ & Friction per actin-substrate bound integrin & 0.02 & $\mathrm{~Pa} \cdot \min$ \\
\hline$\Phi_{u}$ & Additional friction associated to the cell body & $\operatorname{Adh} 0 \cdot 6$ & $\mathrm{~Pa} \cdot \min \cdot \mu \mathrm{m}^{-2}$ \\
\hline$L$ & Length of the cell (fragment) & 10 & $\mu \mathrm{m}$ \\
\hline
\end{tabular}

Table 2: List of parameters for the 1-D simulations, if not otherwise specified. 
prehensive 3-dimensional whole-cell model. From the 1-D and 2-D simulation results we conclude that even without organizing centers (as the cell nucleus or microtubuli, cf. [31]) or regulating systems (as the Rho/Rac control cascades, cf. [54]) the F-actin cytoskeleton and its associated proteins having mechanical functions (myosin and integrin) constitute a self-organizing biophysical system with the ability of autonomous polarization and locomotion. The transition form a symmetric, unpolarized stationary state into a polarized migrating state can be mechanically induced or spontaneous due to stochastic or chaotic fluctuations, depending on the (meta-)stability of the stationary state. Clearly, for reproducing the mentioned experimental data, the 2-dimensional system offers a wider spectrum of possibilities, one of which has been elaborated by Kozlov and Mogilner [33. They prove a defined bi-stability between the radially symmetric cell shape and a polarized, circularly indented shape by allowing for different anisotropic organization of the actin-myosin cytoskeleton. In comparison to this, further numerical 'experiments' using our coupled viscous flow-transport-diffusion-reaction system with free boundary should be performed to explore the capability of isotropic continuum models. Though local orientation or alignment of actin filaments will have an enforcing effect onto the dynamics, interactions and feedback between F-actin, cross-linkers, motor-proteins and adhesion complexes (for a 1-dimensional model see [21]), already the presented simulation results show that internal concentration gradients and directional flow, leading to persistent polarization, can emerge from local fluctuations. Thereby super-threshold pattern formation arises, not as in common excitable media by a purely reaction-diffusion feedback, rather by a spatially distributed coupling between chemically induced force generation/relaxation and mechanically induced bond formation/dissociation.

More than one decade ago, Bereiter-Hahn and Lüers [8] claimed that, based on their experiments and measurements with migrating keratocytes, polarization and directionality of cells are determined by local variations in actin network stiffness and hydrostatic pressure. Together with the more recent high-resolution measurements of dynamic vector field (as F-actin velocity, forces onto pliable substrates, or directionality of FA shape deformations), it could soon be possible to probe and evaluate the various assumptions and hypotheses on mass and force balance conditions, which we postulate in Sections 3.1 and 3.2 . For example, the biphasic correlations between retrograde actin flow speed and traction stress detected by Gardel et al. 222] could well be resolved by the measured and simulated FA gradient away from an advancing lamellar tip, and by our central modeling assumption that the local traction force $\mathbf{F}_{v}$ is proportional to the product $c_{s a} \cdot \mathbf{v}_{c}$ of FA concentration and cortical F-actin velocity.

Moreover, the distribution of flow and force, extensively discussed for the 1-dimensional case (in Section 4.2), has to be quantitatively characterized for the 2-dimensional free boundary situation (Section 4.1), where the overall picture is the same: Wider distribution of traction forces in the leading front region (with maximum flow speed at the tip, depending on the active polymerization pressure) and strong disruptive counter-forces in a confined region of 'rear release'. The actin flow patterns of our 2-D simulations show in 
principle the same characteristics as experimental data by Yam et al. 64 for fish keratocytes. During the process of polarization, the simulated cell looses its radial symmetry of a uniform inward flow pattern, leading to a stronger inward flow in the rear region, whereby the most pronounced forces come from the two sides relative to the establishing locomotion direction, see Fig. 6(c). Indeed, traction force experiments on polarized fish keratocytes have revealed the same pattern, with major traction forces from the two flanks at the rear and weaker retrograde forces at the front [50].

Further evaluation of the presented model should compare the simulated integrin concentration profiles with more precise quantitative data on the temporal turnover and spatial distribution of FAs in migrating cells, as already mentioned above. For this reason, the model has to be extended to include stochastic clustering dynamics of integrins, which probably is an essential feature of FAs determining the detailed patterns of F-actin flow and traction forces. We emphasize, that our model results do not depend on the specific mechanisms of myosin-II contraction. Essential for the emergence of coupled flow and mass gradients is the bimodal (cubic-like) stress function $S=S(\theta)$ in Fig. 5 which similarly appears in a modified mechanical situations as, for example, the nematode sperm motility system, see [9].

The experimental response curves for the migration speed of polarized cells depending on adhesiveness [52] show a broad optimum range with a logarithmic slow decay for larger Adh0 values and a steep decay for lower ones, similar to the curves found in our 1-D model, Fig. 10, and in earlier analogous simulation results using alternative visco-elastic mechanics [17, 24]. The deviating behavior for very low adhesiveness values hat still to be investigated with a more precise quantification of other (weaker) substrate-mediated forces that may come into account.

An optimum curve for the protrusion speed in dependence of the barbed end density $B$, thus also of the concentration Arp0, has been obtained by Mogilner and EdelsteinKeshet [42] with the aid of 1-dimensional diffusion-reaction-transport systems, including membrane resistance and the brownian ratchet mechanism. However, they do not model retrograde actin flow or force transduction to the substratum, so that the shape of their curve ([42],Fig. 6) essentially differs from our results in Figs. 10(b) and 12(b).

Finally, in generalization of the model simulations by Stéphanou et al. [59] and in comparison to other modeling approaches mentioned before, our full 2-dimensional model should be used for reproducing the experimentally observed translocation paths and deformation structures of single blood or tissue cells in culture, which show well-expressed phases of persistent polarization and locomotion, interrupted by events of speed reduction, contractile rounding, successive re-polarization and migration. Statistical analysis, in the same spirit as having been performed for the original annual lamella model [4, could help to reveal the microscopic mechanisms that are responsible for cell motility behavior on the macroscopic level. 
Acknowledgments: This joint work has been generously supported by a grant on "Simulation models for cell motility - coupling substrate adhesion and cytoplasm dynamics" (I/80543, Volkswagen-Stiftung). Moreover, the hospitality of the Soft Matter Physics group at Leipzig University during the final preparation of this chapter is highly appreciated. Finally we thank our colleagues at Bonn University and the Research Center Jülich for helpful discussions and critical remarks.

\section{References}

[1] W. Alt. Biomechanics of actomyosin-dependent mobility of keratinocytes. Biofizika, 41:169-177, 1996.

[2] W. Alt. Nonlinear hyperbolic systems of generalized Navier-Stokes type for interactive motion in biology. In S. Hildebrandt and H. Karcher, editors, Geometric Analysis and Nonlinear Partial Differential Equations, pages 431-461. Springer, 2003.

[3] W. Alt and M. Dembo. Cytoplasm dynamics and cell motion: two-phase flow models. Math. Biosci., 156:207-228, 1999.

[4] W. Alt and R. Tranquillo. Basic morphogenetic system modeling shape changes of migrating cells: how to explain fluctuating lamellipocial dynamics. J. Biol. Syst., 3:905-916, 1995.

[5] W. Alt and R. Tranquillo. Protrusion-retraction dynamics of an annular lamellipodial seam. In W. Alt, A. Deutsch, and G. Dunn, editors, Dynamics of Cell and Tissue Motion, pages 73-81. Birkhäuser, 1997.

[6] D. Ambrosi, A. Duperray, V. Peschetola, and C. Verdier. Traction patterns of tumor cells. J. Math. Biol., 58:163-181, 2009.

[7] G.I. Bell. Models for the specific adhesion of cells to cells. Science, 200:618-627, 1978.

[8] J. Bereiter-Hahn and H. Lüers. Subcellular tension fields and mechanical resistance of the lamella front related to the direction of locomotion. Cell Biochem. Biophys., 29:243-262, 1998.

[9] D. Bottino, A. Mogilner, T. Roberts, M. Stewart, and G. Oster. How nematode sperm crawl. J. Cell. Sci., 115:367-384, 2002.

[10] K. Burridge, M. Chrzanowska-Wodnicka, and C. Zhong. Focal adhesion assembly. Trends Cell Biol., 7:342-347, 1997.

[11] M. Dembo. The mechanics of motility in dissociated cytoplasm. Biophys. J., 50:1165$1183,1986$. 
[12] M. Dembo. Field theories of the cytoplasm. Comments Theor. Biol., 1:159, 1989.

[13] M. Dembo and F.W. Harlow. Cell Motion, contractile networks, and the physics of penetrating reactive flow. Biophys. J., 50:109-121, 1986.

[14] M. Dembo, F.W. Harlow, and W. Alt. The biophysics of cell surface mobility. In A.D. Perelson, Ch. DeLisi, and F.W. Wiegel, editors, Cell Surface Dynamics, Concepts and Methods, pages 495-542. Marcel Dekker, 1984.

[15] M. Dembo and Y.L. Wang. Stresses at the cell-to-substrate interface during locomotion of fibroblasts. Biophys. J., 76:2307-2316, 1999.

[16] R.B. Dickinson. Models for actin polymerization motors. J. Math. Biol., 58:81-103, 2009 .

[17] P.A. DiMilla, K. Barbee, and D.A. Lauffenburger. Mathematical model for the effects of adhesion and mechanics on cell migration speed. Biophys. J., 60:15-37, 1991.

[18] E. Evans and A. Yeung. Apparent viscosity and cortical tension of blood granulocytes determined by micropipet aspiration. Biophys. J., 56:151-160, 1989.

[19] W. Feneberg, M. Westphal, and E. Sackmann. Dictyostelim cells' cytoplasm as an active viscoplastic body. Eur. Biophys. J., 30:284-294, 2001.

[20] D.E. Frank and W.G. Carter. Laminin 5 deposition regulates keratinocyte polarization and persistent migration. J. Cell. Sci., 117:1351-1363, 2004.

[21] J. Fuhrmann, J. Käs, and A. Stevens. Initiation of cytoskeletal asymmetry for cell polarization and movement. J. Theor. Biol., 249:278-288, 2007.

[22] M.L. Gardel, B. Sabass, L. Ji, G. Danuser, U.S. Schwarz, and C.M. Waterman. Traction stress in focal adhesions correlates biphasically with actin retrograde flow speed. J. Cell Biol., 183:999-1005, 2008.

[23] A. Gholami, , M. Falcke, and E. Frey. Velocity oscillations in actin-based motility. New J. Phys., 10:033022 (12pp), 2008.

[24] M.E. Gracheva and H.G. Othmer. A continuum model of motility in ameboid cells. Bull. Math. Biol., 66:167-193, 2004.

[25] H.P. Grimm, A.B. Verkhovsky, A. Mogilner, and J.J. Meister. Analysis of actin dynamics at the leading edge of crawling cells: implications for the shape of keratocyte lamellipodia. Eur. Biophys. J., 32:563-577, 2003.

[26] M. Herant, W.A. Marganski, and M. Dembo. The mechanics of neutrophils: synthetic modeling of three experiments. Biophys. J., 84:3389-3413, 2003. 
[27] B. Hinz, W. Alt, C. Johnen, V. Herzog, and H.W. Kaiser. Quantifying lamella dynamics of cultured cells by SACED, a new computer-assisted motion analysis. Exp. Cell Res., 251:234-243, 1999.

[28] F. Huber, J. Käs, and B. Stuhrmann. Growing actin networks form lammelipodium and lamellum by self-assembly. Biophys. J., 95:5508, 2008.

[29] G. Jiang, G. Giannone, D.R. Critchley, E. Fukumoto, and M.P. Sheetz. Twopiconewton slip bond between fibronectin and the cytoskeleton depends on talin. $\mathrm{Na}$ ture, 424:334-337, 2003.

[30] X. Jiang, D.A. Bruzewicz, A.P. Wong, M. Piel, and G.M. Whitesides. Directing cell migration with asymmetric micropatterns. PNAS, 102:975-978, 2005.

[31] I. Kaverina, O. Krylyshkina, and J.V. Small. Regulation of substrate adhesion dynamics during cell motility. Int. J. Biochem. Cell Biol., 34:746-761, 2002.

[32] S.A. Koestler, S. Auinger, M. Vinzenz, K. Rottner, and J.V. Small. Differentially oriented populations of actin filaments generated in lamellipodia collaborate in pushing and pausing at the cell front. Nat. Cell Biol., 10:306-313, 2008.

[33] M.M. Kozlov and A. Mogilner. Model of polarization and bistability of cell fragments. Biophys. J., 93:3811-3819, 2007.

[34] K. Kruse, J.F. Joanny, F. Jülicher, and J. Prost. Contractility and retrograde flow in lammelipodium motion. Phys. Biol., 3:130-137, 2006.

[35] E. Kuusela and W. Alt. Continuum model of cell adhesion and migration. J. Math. Biol., 58:135-161, 2009.

[36] D.A. Lauffenburger and A.F. Horwitz. Cell migration: A physically integrated molecular process. Cell, 84:359-369, 1996.

[37] T. Libotte, H.W. Kaiser, W. Alt, and T. Bretschneider. Polarity, protrusion-retraction dynamics and their interplay during keratinocyte cell migration. Exp. Cell Res., 270:129-137, 2001.

[38] C.M. Lo, H.B. Wang, M. Dembo, Y.L. Wang, and Y.L. Wang. Cell movement is guided by the rigidity of the substrate. Biophys. J., 79:144-152, 2000.

[39] A.F. Marée, A. Jilkine, A. Dawes, V.A. Grieneisen, and L. Edelstein-Keshet. Polarization and movement of keratocytes: a multiscale modelling approach. Bull. Math. Biol., 68:1169-1211, 2006.

[40] R. Merkel, N. Kirchgessner, C.M. Cesa, and B. Hoffmann. Cell force microscopy on elastic layers of finite thickness. Biophys. J., 93:3314-3323, 2007. 
[41] A. Mogilner. Mathematics of cell motility: have we got its number? J. Math. Biol., 58:105-134, 2009.

[42] A. Mogilner and L. Edelstein-Keshet. Regulation of actin dynamics in rapidly moving cells: a quantitative analysis. Biophys. J., 83:1237-1258, 2002.

[43] A. Mogilner and G. Oster. Cell motility driven by actin polymerization. Biophys. J., 71:3030-3045, 1996.

[44] A. Mogilner and G. Oster. Polymer motors: pushing out the front and pulling up the back. Curr. Biol., 13:R721-733, 2003.

[45] C. Möhl. Modellierung von Adhäsions- und Cytoskelett-Dynamik in Lamellipodien migratorischer Zellen. Diploma thesis, Universität Bonn, Germany, 2005.

[46] S.I. Nishimura and M. Sasai. Modulation of the reaction rate of regulating protein induces large morphological and motional change of amoebic cell. J. Theor. Biol., $245: 230-237,2007$.

[47] S.I. Nishimura, M. Ueda, and M. Sasai. Cortical factor feedback model of cellular locomotion and cytofission. PLoS Comp. Biol., 5(3):e1000310, 2009.

[48] D. Oelz, C. Schmeiser, and A. Soreff. Multistep navigation of leukocytes: a stochastic model with memory effects. Math. Med. Biol., 22:291-303, 2005.

[49] J.M. Oliver, J.R. King, K.J. McKinlay, P.D. Brown, D.M. Grant, C.A. Scotchford, and J.V. Wood. Thin-film theories for two-phase reactive flow models of active cell motion. Math. Med. Biol., 22:53-98, 2005.

[50] T. Oliver, M. Dembo, and K. Jacobson. Separation of propulsive and adhesive traction stresses in locomoting keratocytes. J. Cell Biol., 145:589-604, 1999.

[51] S.P. Palecek, A.F. Horwitz, and D.A. Lauffenburger. Kinetic model for integrinmediated adhesion release during cell migration. Ann. Biomed. Eng., 27:219-235, 1999 .

[52] S.P. Palecek, J.C. Loftus, M.H. Ginsberg, D.A. Lauffenburger, and A.F. Horwitz. Integrin-ligend binding properties govern cell migration speed through cell-substratum adhesiveness. Nature, 385:537-540, 1997.

[53] A. Ponti, M. Machacek, S.L. Gupton, C.M. Waterman-Storer, and G. Danuser. Two distinct actin networks drive the protrusion of migrating cells. Science, 305:1782-1786, 2004 . 
[54] A.J. Ridley, M.A. Schwartz, K. Burridge, R.A. Firtel, M.H. Ginsberg, G. Borisy, J.T. Parsons, and A.R. Horwitz. Cell migration: integrating signals from front to back. Science, 302:1704-1709, 2003.

[55] L. Satish, H.C. Blair, A. Glading, and A. Wells. Interferon-inducible protein 9 (CXCL11)-induced cell motility in keratinocytes requires calcium flux-dependent activation of mu-calpain. Mol. Cell. Biol., 25:1922-1941, 2005.

[56] U.S. Schwarz, N.Q. Balaban, D. Riveline, A. Bershadsky, B. Geiger, and S.A. Safran. Calculation of forces at focal adhesions from elastic substrate data: the effect of localized force and the need for regularization. Biophys. J., 83:1380-1394, 2002.

[57] U. Seifert. Rupture of multiple parallel molecular bonds under dynamic loading. Phys. Rev. Lett., 84:2750-2753, 2000.

[58] J.V. Small, T. Stradal, E. Vignal, and K. Rottner. The lamellipodium: where motility begins. Trends Cell Biol., 12:112-120, 2002.

[59] A. Stéphanou, E. Mylona, M. Chaplain, and P. Tracqui. A computational model of cell migration coupling the growth of focal adhesions with oscillatory cell protrusions. J. Theor. Biol., 253:701-716, 2008.

[60] T.M. Svitkina, A.B. Verkhovsky, K.M. McQuade, and G.G. Borisy. Analysis of the actin-myosin II system in fish epidermal keratocytes: mechanism of cell body translocation. J. Cell Biol., 139:397-415, 1997.

[61] M. Théry, V. Racine, A. Pépin, M. Piel, Y. Chen, J.B. Sibarita, and M. Bornens. The extracellular matrix guides the orientation of the cell division axis. Nat. Cell Biol., 7:947-953, 2005.

[62] C.E. Turner. Paxillin and focal adhesion signalling. Nat. Cell Biol., 2:E231-236, 2000.

[63] A.B. Verkhovsky, T.M. Svitkina, and G.G. Borisy. Self-polarization and directional motility of cytoplasm. Curr. Biol., 9:11-20, 1999.

[64] P.T. Yam, C.A. Wilson, L. Ji, B. Hebert, E.L. Barnhart, N.A. Dye, P.W. Wiseman, G. Danuser, and J.A. Theriot. Actin-myosin network reorganization breaks symmetry at the cell rear to spontaneously initiate polarized cell motility. J. Cell Biol., 178:1207$1221,2007$.

[65] C. Zhu and R. Skalak. A continuum model of protrusion of pseudopod in leukocytes. Biophys. J., 54:1115-1137, 1988. 